\title{
CD28/CTLA-4/ICOS haplotypes confers susceptibility to Graves' disease and modulates clinical phenotype of disease
}

\author{
Edyta Pawlak-Adamska ${ }^{1}$ - Irena Frydecka ${ }^{1}$ Marek Bolanowski ${ }^{2} \cdot$ \\ Anna Tomkiewicz ${ }^{1}$ Anna Jonkisz ${ }^{3}$. Lidia Karabon ${ }^{1}$ - Anna Partyka ${ }^{1}$. \\ Oskar Nowak ${ }^{4} \cdot$ Marek Szalinski $^{5} \cdot$ Jacek Daroszewski $^{2}$
}

Received: 8 April 2016 / Accepted: 17 August 2016 / Published online: 16 September 2016

(C) Springer Science+Business Media New York 2016

\begin{abstract}
Graves' disease, an autoimmune disease with heterogeneous symptoms including Graves' orbitopathy, has a combined genetic/environmental background, where variations within $C D 28 / C T L A-4 / I C O S$ genes are considered as disease markers.

Association of $C D 28 \mathrm{c} .17+3 \mathrm{~T}>\mathrm{C}(\mathrm{rs} 3116496), C T L A-4 \mathrm{~g}$. 319C $>\mathrm{T}(\mathrm{rs} 5742909), C T L A-4 \mathrm{c} .49 \mathrm{~A}>\mathrm{G}(\mathrm{rs} 231775)$, CTLA4g.*642AT(8_33), CT60(rs3087243), Jo31(rs11571302), ICOSc.1554+4GT(8_15) polymorphisms with susceptibility to Graves' disease and clinical outcome was investigated. The study group comprised of 561 Polish Caucasians, including 172 unrelated Graves' disease patients. CTLA4c.49A > G, CTLA-4g.319C > T, and CT60 were genotyped
\end{abstract}

Electronic supplementary material The online version of this article (doi:10.1007/s12020-016-1096-1) contains supplementary material, which is available to authorized users.

Edyta Pawlak-Adamska

epawlak@iitd.pan.wroc.pl

1 Department of Experimental Therapy, Hirszfeld Institute of Immunology and Experimental Therapy, Polish Academy of Sciences, R. Weigl 12, Wroclaw 53-114, Poland

2 Department of Endocrinology, Diabetes and Isotope Therapy, Wroclaw Medical University, L. Pasteur 4, Wroclaw 50-367, Poland

3 Department of Forensic Medicine, Wroclaw Medical University, M. Curie-Sklodowska 52, Wroclaw 50-369, Poland

4 Department of Human Evolutionary Biology, Institute of Anthropology, Adam Mickiewicz University, Umultowska 89, Poznan 61-614, Poland

5 Department of Ophthalmology, Wroclaw Medical University, Borowska 213, Wroclaw 50-556, Poland by PCR-RFLP; Jo31 and $C D 28 \mathrm{c} .17+3 \mathrm{C}>\mathrm{T}$ by minisequencing; CTLA-4g.*642AT(8_33) and ICOSc.1554+ 4GT(8_15)_PCR and fluorescence-based technique. CD28c.17+3T >C(rs3116496)T/CTLA-4g.319C >T(rs57429 09)C/CTLA-4c.49A $>$ G(rs231775)G/CTLA-4g.*642AT(8_33) $\left(\mathrm{AT}_{16-21}\right) / \mathrm{CT} 60(\mathrm{rs} 3087243) \mathrm{G} / \mathrm{Jo} 31(\mathrm{rs} 11571302) \mathrm{G} / I C O S \mathrm{c}$. $1554+4 \mathrm{GT}\left(8 \_15\right)(\mathrm{m})$ and $\mathrm{TCA}\left(\mathrm{AT}_{<16}\right) \mathrm{GT}(\mathrm{m})$ haplotypes increased risk of Graves' disease, especially in males, as well as overall Graves' orbitopathy development with severe outcome. TCG(AT $\left.{ }_{16-21}\right) \mathrm{GG}(\mathrm{l})$ haplotype increased risk of Graves' disease and reduced the chance of successful medical treatment. Although this haplotype was mainly observed in patients without signs of Graves' orbitopathy, if Graves' orbitopathy developed it favored a Graves' orbi-

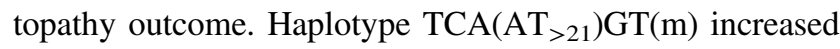
Graves' disease risk in women and, in all patients, was linked to Graves' disease without Graves' orbitopathy. TCG $\left(\mathrm{AT}_{<16}\right) \mathrm{GG}(\mathrm{m})$ haplotype was predominantly observed in patients without Graves' orbitopathy, whereas TCA $\left(\mathrm{AT}_{16-21}\right) \mathrm{GG}(\mathrm{m})$ was absent in those patients. TCA $\left(\mathrm{AT}_{16-21}\right) \mathrm{GG}(\mathrm{m})$ occurred in patients with a mild Graves' orbitopathy outcome. The marker CTLA-4g.*642AT(8_33) was the only independent Graves' disease risk factor, whereas CT60 was an independent factor for disease progression. Sporadic Graves' disease was related to presence of $C T L A-4 \mathrm{c} .49 \mathrm{~A}>\mathrm{G}[\mathrm{A}]$ and the rare $C T L A-4 \mathrm{~g} .319 \mathrm{C}>\mathrm{T}[\mathrm{T}]$ allele variant. Familial background of the disease was exclusively associated with CTLA-4g.*642AT(8_33) $\left[\mathrm{AT}_{>21}\right] /\left[\mathrm{AT}_{>21}\right]$ genotype. CD28/CTLA-4/ICOS loci may confer inherited susceptibility to Graves' disease or may be involved in susceptibility to Graves' disease and play a pathogenetic role.

Keywords CD28/CTLA-4/ICOS · Graves' disease · Graves' orbitopathy $\cdot$ Gene polymorphism $\cdot$ Haplotype 


\section{Introduction}

The pathogenesis of Graves' disease (GD) requires a specific sequence of events, involving both genetic factors and the ensuing environmental factors, for the final clinical phenotype to manifest [1-3]. The orbital inflammatory process in Graves' orbitopathy (GO) is believed to be driven by $\mathrm{T}$ cells in response to thyroid-orbit shared antigens accessing and infiltrating the orbital space [4]. As T cells play a key role in the pathogenesis of GO, and CD28/CTLA4/ICOS co-stimulatory molecules are critical in T-cell activation, they may be considered to be candidate risk factors. Disruption to the precise balance of expression of costimulatory and inhibitory molecules is known to play a role in the pathogenesis of autoimmune diseases [5]. This is supported by studies demonstrating that adolescents and children newly diagnosed with GD had an abnormal percentage of lymphocytes expressing co-stimulatory molecules [6,7]. Similarly, differences in the expression pattern of CD28 in T cells of the thyroid gland and peripheral blood T lymphocytes was observed [6]. Differential regulation of these molecules could easily affect T-cell function and hence the regulation of the immune response. Abnormal expression and/or dysfunction of these molecules may be caused by polymorphisms in the corresponding gene [8-26]. In previous work, we linked the presence of guanine $(\mathrm{G})$ in CT60 and Jo31 single-nucleotide polymorphism (SNPs), located within the 3'UTR of CTLA-4, with an elevated level of the soluble CTLA-4 isoform in GD, especially in cases of severe outcome [26]. Competitive binding of sCTLA-4 with CTLA-4 common ligands inhibited T-cell proliferation via increased activation of CD28 [27]. The prevalence of sCTLA-4-bearing genetics in GD may indicate abnormal expression of CTLA-4 on T-regulatory cells (Treg). In turn, this may affect the precise balance of suppressive and effector activity of Treg, which is strongly linked to pathogenesis of autoimmune diseases [27].

A previously published, candidate gene strategy indicated and validated several immune-related and thyroidspecific susceptibility genes for GD. In regard to immunerelated genes, comprehensive fine mapping of the $C D 28 /$ CTLA-4/ICOS region showed an association signal within a 6.1-Kb 3' region of CTLA-4 [18]. To date, most research has focused on univariate association studies of single polymorphisms - especially the highly polymorphic CTLA4 [8, 9, 16, 18, 21, 25, 28, 29, 30, 31, 32, 33, 34, 35, 36], with several functional polymorphisms having been observed within this gene [8-26]. Similar findings concerning GO and CTLA-4 polymorphisms have been reported [37]. In the case of the co-stimulatory receptors CD28 and ICOS, definitive determination of their role in GD and GO pathogenesis is still impossible due to the paucity of research published to date. In our opinion, treating the $2 \mathrm{q} 33$ chromosomal region where $C D 28, C T L A$ 4, and ICOS are located as a locus is more appropriate and takes into account the possible effects of interactions between polymorphisms located within those genes on their function and/or expression and, consequently, on the pathogenesis and clinical outcome of GD. Such a relationship was previously observed between genetic variants in ICOSIVS1+173T/C and CTLA-4 mRNA expression levels [38].

Based on the concept that the associations of GD and GO with this region may represent the effects of any of these three genes alone, or in combination, due to linkage disequilibrium (LD), we attempted to verify the possible associations between $C D 28 \mathrm{c} .17+3 \mathrm{~T}>\mathrm{C}(\mathrm{rs} 3116496) / C T L A-$ 4g.319C $>\mathrm{T}(\mathrm{rs} 5742909) / C T L A-4 \mathrm{c} .49 \mathrm{~A}>\mathrm{G}(\mathrm{rs} 231775) / C T L A$ -4g.*642AT(8_33)(AT $16-21) / C T 60(r s 3087243) / J o 31(r s 1157$ 1302)/ICOSc.1554+4GT(8_15) gene polymorphisms and susceptibility to GD, as well as the severity and activity of GO defined as one susceptible locus, expressed as the haplotype.

\section{Materials and methods}

\section{Study population}

\section{Patients}

One hundred and seventy-two unrelated Polish Caucasian GD patients consecutively admitted to the tertiary care academic medical center were enrolled into the study. The initial diagnosis of GD was confirmed by routine clinical and laboratory tests. Detailed patient characteristics are presented in Table 1. Ninety-six patients responded to firstline medical treatment and required no further therapy for relapse within next 18 months.

\section{Assessment of ocular changes}

Ophthalmological examination was performed at the time of blood collection. The severity and activity of orbitopathy was assessed using criteria proposed by Bartalena [39] (detailed in Table 1).

\section{Control group}

The control group consisted of 389 apparently healthy volunteers from the same geographical area (225 (57.8\%) females and $164(42.2 \%)$ males). 
Table 1 GD patients $(n=172)$ clinical characteristics

\begin{tabular}{|c|c|c|c|c|}
\hline & \multicolumn{3}{|l|}{ Parameter } & \multirow{2}{*}{$\frac{n(\%)}{28(16.28)}$} \\
\hline Without GO $(n=28)$ & \multicolumn{2}{|c|}{ CAS + orbitopathy index } & 0 & \\
\hline \multirow{11}{*}{ With GO $(n=144)$} & \multirow{8}{*}{ CAS } & \multirow{4}{*}{ Non-active $(n=73)$} & 0 & $10(5.81)$ \\
\hline & & & 1 & $10(5.81)$ \\
\hline & & & 2 & $21(12.21)$ \\
\hline & & & 3 & $32(18.60)$ \\
\hline & & \multirow[t]{4}{*}{ Active $(n=71)$} & 4 & $32(18.60)$ \\
\hline & & & 5 & $21(12.21)$ \\
\hline & & & 6 & $14(8.15)$ \\
\hline & & & 7 & $4(2.33)$ \\
\hline & \multirow[t]{3}{*}{ Orbitopathy index } & \multirow[t]{2}{*}{ Non-severe $(n=64)$} & Mild & $29(16.86)$ \\
\hline & & & Moderate & $35(20.35)$ \\
\hline & & \multicolumn{2}{|c|}{ Severe } & $80(46.50)$ \\
\hline Gender & & \multicolumn{2}{|c|}{ Female/male } & $134(77.90) / 38(22.10)$ \\
\hline Familial autoimmune thyroid history & & \multicolumn{2}{|c|}{ Yes/no } & $138(80.23) / 34(19.77)$ \\
\hline \multirow[t]{5}{*}{ Smoking status } & & Non-smokers $(n=65)$ & Never & $65(37.79)$ \\
\hline & & Smokers $(n=107)$ & In past & $38(22.09)$ \\
\hline & & & $\leq 10$ & $40(23.26)$ \\
\hline & & & $10-20$ & $25(14.53)$ \\
\hline & & & $\geq 20$ & $4(2.33)$ \\
\hline \multirow[t]{3}{*}{ Response to anti-thyroid treatment } & & \multicolumn{2}{|c|}{ Yes } & $96(55.82)$ \\
\hline & & No $(n=76)$ & Thyroidectomy & $16(9.30)$ \\
\hline & & & ${ }^{131}$ I therapy & $60(34.88)$ \\
\hline \multirow[t]{3}{*}{ Age } & & \multicolumn{2}{|l|}{ Mean \pm SD } & $49 \pm 12.73$ \\
\hline & & \multicolumn{2}{|l|}{ Median } & 48 \\
\hline & & \multicolumn{2}{|l|}{ Range } & $23-82$ \\
\hline
\end{tabular}

\section{Ethical approvals}

The local ethics committee approved the study protocol.

\section{Genotyping of polymorphisms}

The CTLA-4g.319C $>\mathrm{T}$ (rs5742909), CTLA-4c.49A $>\mathrm{G}$ (rs231775), CT60 (CTLA-4g.*6230G >A, rs3087243), and Jo31 (CTLA-4g.*10223G>T, rs11571302) polymorphisms were examined as described by Daroszewski et al. [26].

The $C D 28 \mathrm{c} .17+3 \mathrm{~T}>\mathrm{C}$ SNP (rs3116496, IVS3+17C/T), CTLA-4g.*642AT(8_33) and ICOSc.1554+4GT(8_15) short tandem repeat polymorphisms were genotyped according to Suwalska et al. [40].

\section{Statistical analyses}

The Hardy-Weinberg equilibrium (HWE) was tested for the studied group. Categorical data between GD patients and controls, and within the GD group according to clinical outcome, were compared using the $\chi^{2}$ test with appropriate correction. In case of multiple comparisons, Bonferroni multiple adjustments were employed. Haplotype analysis and LD coefficients were estimated using the SHEsis software (http://202.120.7.14/analysis/myAnalysis.php).

The combined effects of polymorphic markers and environmental factors (gender and smoking habits) on the development of GD and GD outcome were analyzed by unconditional multivariate logistic regression analysis with a logit regression model using a quasi-Newton estimation method (software EPIINFO Ver. 7.1.1.14).

Differences were considered statistically significant if the $p$-value $<0.05$.

\section{Results}

Genotype frequencies in both groups were in HWE (Table 2).

For CTLA-4g.*642AT(8_33) polymorphism, 20 alleles and 90 genotypes were identified (data not shown). Alleles were grouped into three groups for further analysis: $\left[\mathrm{AT}_{<16}\right]$ with less than $16,\left[\mathrm{AT}_{16-21}\right]$ with $16-21$, and $\left[\mathrm{AT}_{>21}\right]$ with more than 21 AT repeats. 
Table 2 CTLA-4g.*642AT(8_32), CT60 (rs3087243, CTLA-4g.*6230G>A), and ICOSc.1554+4GT(8_15) genotype and allele frequencies in GD patients and control group

$\begin{array}{lllllll}\begin{array}{l}\text { GD patients } \\ n(\%)\end{array} & \begin{array}{l}\text { Control } \\ \text { group } n(\%)\end{array} & p_{\text {global }}{ }^{*} & \chi^{2} & p & \text { Odds ratio } & \begin{array}{l}95 \% \\ \text { confidence } \\ \text { interval }\end{array}\end{array}$

CTLA-4g.*642AT(8_33)

Genotype $^{a}$

\begin{tabular}{|c|c|c|c|c|c|c|c|}
\hline$\left[\mathrm{AT}_{<16}\right] /\left[\mathrm{AT}_{<16}\right]$ & $31(18.0)$ & 99 (25.4) & $0.08^{\wedge}$ & Referent & & & \\
\hline$\left[\mathrm{AT}_{<16}\right] /\left[\mathrm{AT}_{16-21}\right]$ & $52(30.2)$ & $104(26.7)$ & & 3.10 & 0.08 & 1.60 & $0.95-2.69$ \\
\hline$\left[\mathrm{AT}_{<16}\right] /\left[\mathrm{AT}_{>21}\right]$ & $22(12.8)$ & $64(16.5)$ & & 0.08 & 0.77 & 1.10 & $0.58-2.06$ \\
\hline$\left[\mathrm{AT}_{16-21}\right] /\left[\mathrm{AT}_{16-21}\right]$ & 35 (20.3) & $50(12.9)$ & & 7.26 & 0.007 & 2.24 & $1.24-4.04$ \\
\hline$\left[\mathrm{AT}_{16-21}\right] /\left[\mathrm{AT}_{>21}\right]$ & 30 (17.4) & 54 (13.9) & & 3.53 & 0.06 & 1.77 & $0.97-3.24$ \\
\hline$\left[\mathrm{AT}_{>21}\right] /\left[\mathrm{AT}_{>21}\right]$ & $2(1.2)$ & $18(4.6)$ & & $1.21 *$ & $0.27 *$ & 0.35 & $0.08-1.62$ \\
\hline \multicolumn{8}{|l|}{ Allele $^{b}$} \\
\hline$\left[\mathrm{AT}_{<16}\right]$ & $136(39.5)$ & $366(47.0)$ & $0.006^{\wedge}$ & Referent & & & \\
\hline$\left[\mathrm{AT}_{16-21}\right]$ & $152(44.2)$ & $258(33.2)$ & & 10.41 & 0.001 & 1.59 & $1.20-2.10$ \\
\hline$\left[\mathrm{AT}_{>21}\right]$ & $56(16.3)$ & $154(19.8)$ & & 0.01 & 1.00 & 0.98 & $0.68-1.41$ \\
\hline \multicolumn{8}{|l|}{ Dominant model } \\
\hline \multirow[t]{2}{*}[\mathrm{AT}_{<16}]{$/\left[\mathrm{AT}_{16-21}\right]+\left[\mathrm{AT}_{16-21}\right] /\left[\mathrm{AT}_{16-21}\right]+\left[\mathrm{AT}_{16-21}\right] /\left[\mathrm{AT}_{>21}\right] v s$. others } & $117(68.0)$ & $208(53.5)$ & & 10.36 & 0.001 & 1.85 & $1.27-2.70$ \\
\hline & $55(32.0)$ & $181(46.5)$ & & & & 0.54 & $0.37-0.79$ \\
\hline \multicolumn{8}{|l|}{ CT60 (CTLA-4g.*6230G>A, rs3087243) } \\
\hline \multicolumn{8}{|l|}{ Genotype $^{c}$} \\
\hline$[\mathrm{GG}]$ & $74(43.0)$ & $133(34.3)$ & $0.08^{\wedge}$ & Referent & & & \\
\hline$[\mathrm{GA}]$ & $80(46.5)$ & $187(48.2)$ & & 1.78 & 0.18 & 0.77 & $0.52-1.13$ \\
\hline$[\mathrm{AA}]$ & $18(10.5)$ & $68(17.5)$ & & 6.19 & 0.01 & 0.48 & $0.26-0.86$ \\
\hline \multicolumn{8}{|l|}{ Allele } \\
\hline$[\mathrm{G}]$ & $228(66.3)$ & $453(58.4)$ & & 6.09 & 0.01 & 1.41 & $1.08-1.84$ \\
\hline [A] & $116(33.7)$ & $323(41.6)$ & & & & 0.72 & $0.55-0.93$ \\
\hline \multicolumn{8}{|l|}{ Dominant model } \\
\hline \multirow[t]{2}{*}[\mathrm{GG}]{$+[\mathrm{GA}] v s .[\mathrm{AA}]$} & $154(89.7)$ & $320(82.5)$ & & 4.57 & 0.03 & 1.82 & $1.05-3.16$ \\
\hline & $18(10.3)$ & $68(17.5)$ & & & & 0.55 & $0.32-0.96$ \\
\hline \multicolumn{8}{|l|}{ Recessive model } \\
\hline \multirow[t]{2}{*}[\mathrm{AA}]{$+[\mathrm{GA}] v s .[\mathrm{GG}]$} & $98(57.0)$ & $255(65.7)$ & & 3.91 & 0.05 & 0.69 & $0.48-1.00$ \\
\hline & $74(43.0)$ & $134(34.4)$ & & & & 1.45 & $1.00-2.09$ \\
\hline \multicolumn{8}{|l|}{ ICOSc.1554+4GT(8_15) } \\
\hline \multicolumn{8}{|l|}{ Genotype $^{d}$} \\
\hline$(\mathrm{s}) /(\mathrm{s})$ & $1(0.6)$ & $4(1.0)$ & $\mathrm{ns}^{\wedge}$ & $0.00^{*}$ & $1.00^{*}$ & 0.58 & $0.06-5.29$ \\
\hline$(\mathrm{s}) /(\mathrm{m})$ & $5(2.9)$ & $35(9.0)$ & & 4.46* & $0.03 *$ & 0.33 & $0.13-0.88$ \\
\hline (s)/(1) & $7(4.1)$ & $17(4.4)$ & & 0.009 & 1.00 & 0.96 & $0.38-2.41$ \\
\hline$(\mathrm{m}) /(\mathrm{m})$ & $71(41.3)$ & $166(42.9)$ & & Referent & & & \\
\hline$(\mathrm{m}) /(\mathrm{l})$ & 77 (44.8) & $137(35.4)$ & & 1.77 & 0.18 & 1.31 & $0.88-1.94$ \\
\hline (1)/(1) & $11(6.4)$ & $28(7.2)$ & & 0.06 & 0.81 & 1.10 & $0.52-2.32$ \\
\hline \multicolumn{8}{|l|}{ Allele $^{e}$} \\
\hline (s) & $14(4.1)$ & $60(7.8)$ & $\mathrm{ns}^{\wedge}$ & 4.58 & 0.03 & 0.52 & $0.29-0.96$ \\
\hline (m) & $224(65.1)$ & $504(65.1)$ & & Referent & & & \\
\hline (l) & $106(30.8)$ & $210(27.1)$ & & 0.74 & 0.39 & 0.88 & $0.67-1.17$ \\
\hline \multicolumn{8}{|l|}{ Dominant model } \\
\hline \multirow[t]{2}{*}{$(\mathrm{m}) /(\mathrm{m})+(\mathrm{m}) /(\mathrm{l}) v s$. others } & $153(89.0)$ & $338(87.3)$ & & 0.29 & 0.59 & 0.86 & $0.49-1.50$ \\
\hline & $19(11.0)$ & $49(12.7)$ & & & & 1.17 & $0.67-2.05$ \\
\hline
\end{tabular}

Bold indicates statistical significant association

ICOSc.1554+4GT(8_15): (s)-short alleles with 8 and 9 repeats of GT pair; (m)-medium alleles with 10 and 11 repeats of GT pair; (1)-long alleles with 12 or more repeats of GT pair

OR odds ratio, $95 \%$ CI $95 \%$ confidence intervals, HWE Hardy-Weinberg equilibrium

$\wedge^{\wedge}$ - $p$-value after Bonferroni correction; *-p-value after Yate's correction

${ }^{\mathrm{a}} \chi^{2}=13.85, \mathrm{df}=5, p^{\prime \prime}=0.02, n_{\mathrm{GDpatients}}=172, n_{\text {controls }}=389$

b $\chi^{2}=12.51, \mathrm{df}=3, p^{\prime \prime}=0.002$

${ }^{\mathrm{c}} \chi^{2}=6.40, \mathrm{df}=2, p^{\prime \prime}=0.04, n_{\mathrm{GDpatients}}=172, \mathrm{HWE}_{\mathrm{GDpatients}}: \chi^{2}=0.28, p=0.60, n_{\text {controls }}=388, \mathrm{HWE}_{\mathrm{controls}}: \chi^{2}=0.03, p=0.87$

${ }^{\mathrm{d}} \chi^{2}=9.46, \mathrm{df}=5, p^{\prime \prime}=0.09, n_{\mathrm{GDpatients}}=172, n_{\text {controls }}=387$

e $\chi^{2}=6.02, \mathrm{df}=3, p^{\prime \prime}=0.05$ 
For ICOSc.1554+4GT(8_15) polymorphism, 8 alleles and 19 genotypes were observed (data not shown); alleles were grouped into three cohorts: short alleles (s) with 8-9, medium (m) with 10-11, and long alleles (l) with 12 or more GT repeats.

Smokers were statistically more prone to severe eye symptoms $(p=0.04)$, and patients with non-severe GO were the best responders to anti-thyroid treatment ( $p=$ 0.001) (Supplementary Table 1).

\section{CD28/CTLA-4/ICOS gene polymorphisms and GD risk}

In univariate analysis a significant association with GD was observed only with two of the studied polymorphisms: g.*642AT(8_33) and CT60 (rs3087243) (Table 2).

The CTLA-4g.*642AT(8_33)[AT $\left.\mathrm{AT}_{16-21}\right]$ variant increased the risk of disease 2.24-fold $(p=0.007,95 \% \mathrm{CI}$ : 1.24-4.04), and the carriers of this allele variant were 1.85 times more prone to GD ( $p=0.001,95 \% \mathrm{CI}: 1.27-2.70)$.

CT60 (rs3087243) [AA] homozygosity significantly protected against GD $(p=0.01, \mathrm{OR}=0.48,95 \% \mathrm{CI}$ : 0.26-0.86). Weaker association was seen for overall presence of this allele $(p=0.03, \mathrm{OR}=0.69,95 \% \mathrm{CI}$ : 0.48-1.00) (Table 2).

Other studied polymorphisms were not associated with GD risk (Supplementary Table 2).

In the controls robust LD was observed between the pair of CTLA-4 polymorphisms, and weaker association was seen between CTLA-4 polymorphisms and CD28c.17 $+3 \mathrm{~T}>\mathrm{C}$ (rs3116496), while in patients such relations were observed only between CTLA-4 gene polymorphisms (Supplementary Table 3).

Significantly different haplotype distribution among GD patients and controls was observed $\left(\chi^{2}=25.028397\right.$, $\left.p_{\text {corrected }}=0.00069\right)$. Haplotypes $\mathrm{TCA}\left[\mathrm{AT}_{<16}\right] \mathrm{AT}(\mathrm{l}), \mathrm{TCG}$ $\left[\mathrm{AT}_{>21}\right] \mathrm{GG}(\mathrm{m}) \quad$ in order: $C D 28 \mathrm{c} .17+3 \mathrm{~T}>\mathrm{C} / \mathrm{g} .319 \mathrm{C}>$ T/c.49A>G/g.*642AT(8_33)/CT60/Jo31/ICOSc.1554+4GT (8_15)] decreased the risk of GD $(p=0.029116$, $\mathrm{OR}=0.499, \quad 95 \% \quad \mathrm{CI}: \quad 0.265-0.941 ; \quad p=0.000456$, $\mathrm{OR}=0.195, \quad 95 \%$ CI: $0.071-0.535)$, whereas TCG $\left[\mathrm{AT}_{16-21}\right] \mathrm{GG}(\mathrm{l})$ and TTA $\left[\mathrm{AT}_{>21}\right] \mathrm{GG}(\mathrm{m})$ amplified such risk $(p=0.053837, \quad$ OR $=1.455, \quad 95 \% \quad$ CI: $0.993-2.133$; $p=0.050925, \quad \mathrm{OR}=2.030, \quad 95 \% \quad \mathrm{CI}: \quad 0.985-4.185)$

(Supplementary Table 4).

\section{CD28/CTLA-4/ICOS gene polymorphisms and gender}

The CTLA-4g.*642AT(8_33)[AT $\left.{ }_{16-21}\right] /\left[\mathrm{AT}_{16-21}\right]$ genotype increased risk of disease in women $(p=0.01, \mathrm{OR}=2.48)$, whereas $\left[\mathrm{AT}_{16-21}\right]$ allele increased the risk of disease in both gender ( $p=0.007 ; p=0.05$ ) (Supplementary Table 5).

Moreover, women possessing the CT60 (rs3087243) [AA] genotype have lower risk of developing GD $(p=0.02$,
$\mathrm{OR}=0.43,95 \% \mathrm{CI}: 0.22-0.86)$, similarly was for the allele and dominant model $(p=0.02 ; p=0.02)$ (Supplementary Table 5).

There also the ICOSc.1554+4GT(8_15)(1) allele increased risk of GD in women $(p=0.003, \mathrm{OR}=1.72,95 \%$ CI: 1.21-2.46) (Supplementary Table 5).

TCG $\left[\mathrm{AT}_{>21}\right] \mathrm{GG}(\mathrm{m})$ haplotype protected against disease after stratification according to gender $(p=0.007, p=$ 0.008). Moreover, $\left.\mathrm{TCA}_{[\mathrm{AT}}{ }_{<16}\right] \mathrm{GT}(\mathrm{m})$ and $\mathrm{CTA}\left[\mathrm{AT}_{16-21}\right]$ GG(1) haplotypes were observed only in male patients, and TCA $\left[\mathrm{AT}_{<16}\right] \mathrm{AG}(\mathrm{m})$ and TCG[AT $\left.{ }_{16-21}\right] \mathrm{GG}(\mathrm{m})$ haplotypes increased disease risk in male $(p=0.03, \mathrm{OR}=5.93,95 \%$ CI: $0.94-37.51 ; p=0.05, \mathrm{OR}=1.93,95 \%$ CI: $1.00-3.74)$. Contrary, haplotypes TCA[AT $\left.\left.{ }_{<16}\right] \mathrm{AT}(\mathrm{l}), \mathrm{TCG} \mathrm{AT}_{>21}\right] \mathrm{GG}$ $(\mathrm{m})$, and $\mathrm{CTA}\left[\mathrm{AT}_{>21}\right] \mathrm{GG}(\mathrm{m})$ were absent in male patients (Supplementary Table 6).

Additionally, stratification by gender showed differed frequency of TCA $\left[\mathrm{AT}_{<16}\right] \mathrm{AG}(\mathrm{m})$ and CTA[AT $\left.{ }_{16-21}\right] \mathrm{GG}(\mathrm{l})$ within GD $(p=0.04, p=0.001)$, whereas within controls this was observed for CTA[AT $\left.{ }_{>21}\right] \mathrm{GG}(\mathrm{m})$ haplotype $(p=$ 0.02) (Supplementary Table 6).

\section{GD risk factors-multivariate associations}

The unconditional stepwise multivariate logistic regression analysis of all studied polymorphisms, including three prone genetic parameters: presence of $\mathrm{T}$ allele at CTLA4g.319C $>$ T (rs5742909) (genotype TT and CT), presence of CTLA-4g.*642AT(8_33)[AT $16-21]$ allele, presence of $\mathrm{G}$ allele at CT60 (rs3087243) [genotype (GG) and (GA)], and environmental factor-female gender, showed that only the CTLA-4g.*642AT(8_33) was an independent GD risk factor $\left(\chi^{2}=33.3, p=0.0008\right)$. In detail, presence of $C T L A-4 \mathrm{~g}$. *642AT(8_33)[AT AT-21 $_{16}$ allele 1.94-fold increased risk for GD $(p=0.00081,95 \%$ CI: 1.32-2.86).

\section{CD28/CTLA-4/ICOS gene polymorphisms and clinical data in GD}

\section{Familial autoimmune thyroid history}

The CTLA-4g.319C>T (rs5742909) genotype and allele were differentially distributed $\left(p=0.0002 ; p=4.07 \times 10^{-5}\right)$, and lack of the rare [T] allele increased GD in patients with familial autoimmune thyroid incidence by 4.85 -fold $(p=$ 0.00005, $95 \%$ CI: 2.18-10.76). CTLA-4g.*642AT(8_33) $\left[\mathrm{AT}_{>21}\right] /\left[\mathrm{AT}_{>21}\right]$ genotype was also present only in patients with familial autoimmune thyroid background $\left(p_{\text {corrected }}=\right.$ 0.04), whereas presence of the CTLA-4c.49A $>\mathrm{G}$ (rs231775) [A] allele variant was significantly associated with sporadic GD $\left(p_{\text {corrected }}=0.05\right)$ (Table 3 and Supplementary Table 7).

A significant difference in haplotype frequency in GD patients with respect to familial thyroid autoimmune history 
Table 3 CTLA-4g.319C>T, CTLA-4c.49A>G, CTLA-4g.*642AT(8_32), ICOSc.1554+4GT(8_15) genotype and allele frequencies in GD patients according to occurrence of familial autoimmune thyroid history in first-line relatives

\begin{tabular}{|c|c|c|c|c|c|c|}
\hline & \multicolumn{2}{|c|}{ Familial autoimmune thyroid history } & \multirow[t]{2}{*}{$\chi^{2}$} & \multirow[t]{2}{*}{$p$-value } & \multirow[t]{2}{*}{ Odds ratio } & \multirow{2}{*}{$\begin{array}{l}95 \% \text { confidence } \\
\text { interval }\end{array}$} \\
\hline & $\begin{array}{l}\text { Without } \\
(n=34) n(\%)\end{array}$ & $\begin{array}{l}\text { With } \\
(n=138) n(\%)\end{array}$ & & & & \\
\hline \multicolumn{7}{|l|}{ CTLA-4 g.319C >T (rs5742909) } \\
\hline \multicolumn{7}{|l|}{ Genotype $^{a}$} \\
\hline$[\mathrm{CC}]$ & $16(47.1)$ & $112(81.2)$ & & & Referent & \\
\hline$[\mathrm{CT}]$ & $16(47.1)$ & $24(17.4)$ & 14.95 & 0.0001 & 0.21 & $0.09-0.49$ \\
\hline [TT] & $2(5.9)$ & $2(1.4)$ & $2.00^{*}$ & $0.16^{*}$ & 0.14 & $0.02-1.09$ \\
\hline \multicolumn{7}{|l|}{ Allele } \\
\hline$[\mathrm{C}]$ & $48(70.6)$ & $248(89.9)$ & 16.87 & $4.07 \times 10^{-5}$ & 3.69 & $1.92-7.08$ \\
\hline$[\mathrm{T}]$ & $20(29.4)$ & $28(10.1)$ & & & 0.27 & $0.14-0.52$ \\
\hline \multicolumn{7}{|l|}{ Dominant model } \\
\hline \multirow[t]{2}{*}[\mathrm{CC}]{$+[\mathrm{CT}]$ vs. $[\mathrm{TT}]$} & $32(94.1)$ & 136 (98.6) & $0.81^{*}$ & $0.37 *$ & 4.25 & $0.58-31.32$ \\
\hline & $2(5.9)$ & $2(1.4)$ & & & 0.24 & $0.03-1.73$ \\
\hline \multicolumn{7}{|l|}{ Recessive model } \\
\hline \multirow[t]{2}{*}[\mathrm{TT}]{$+[\mathrm{CT}]$ vs. $[\mathrm{CC}]$} & $18(52.9)$ & $26(18.8)$ & 16.66 & $4.5 \times 10^{-5}$ & 0.21 & $0.09-0.46$ \\
\hline & $16(47.1)$ & $112(81.2)$ & & & 4.85 & $2.18-10.76$ \\
\hline \multicolumn{7}{|l|}{ CTLA-4c.49A>G (rs231775) } \\
\hline \multicolumn{7}{|l|}{ Genotype $^{b}$} \\
\hline$[\mathrm{AA}]$ & $12(35.3)$ & $39(28.3)$ & & & Referent & \\
\hline$[\mathrm{AG}]$ & $19(55.9)$ & $70(50.7)$ & 0.09 & 0.76 & 0.88 & $0.39-2.01$ \\
\hline$[\mathrm{GG}]$ & $3(8.8)$ & $29(21.0)$ & $1.79 *$ & $0.18^{*}$ & 2.97 & $0.77-11.51$ \\
\hline \multicolumn{7}{|l|}{ Allele } \\
\hline$[\mathrm{A}]$ & $43(63.2)$ & $148(53.6)$ & 2.04 & 0.15 & 0.67 & $0.39-1.16$ \\
\hline$[\mathrm{G}]$ & $25(36.8)$ & $128(46.4)$ & & & 1.49 & $0.86-2.57$ \\
\hline \multicolumn{7}{|l|}{ Dominant model } \\
\hline \multirow[t]{2}{*}[\mathrm{AA}]{$+[\mathrm{AG}] v s .[\mathrm{GG}]$} & $31(91.2)$ & $109(79.0)$ & $3.85^{*}$ & $0.05^{*}$ & 0.27 & $0.08-0.94$ \\
\hline & $3(8.8)$ & $29(21.0)$ & & & 3.70 & $1.07-12.78$ \\
\hline Recessive model & & & & & & \\
\hline$[\mathrm{GG}]+[\mathrm{AG}] v s .[\mathrm{AA}]$ & $22(64.7)$ & $99(71.7)$ & 0.65 & 0.42 & 1.38 & $0.63-3.07$ \\
\hline & $12(35.3)$ & $39(28.3)$ & & & 0.72 & $0.33-1.60$ \\
\hline CTLA-4 g.*642AT(8_33) & & & & & & \\
\hline Genotype $^{c}$ & & & & & & \\
\hline$\left[\mathrm{AT}_{<16}\right] /\left[\mathrm{AT}_{<16}\right]$ & $6(17.6)$ & $25(18.1)$ & & & Referent & \\
\hline$\left[\mathrm{AT}_{<16}\right] /\left[\mathrm{AT}_{16-21}\right]$ & $10(29.4)$ & $42(30.4)$ & 0.00 & 1.00 & 1.01 & $0.33-3.11$ \\
\hline$\left[\mathrm{AT}_{<16}\right] /\left[\mathrm{AT}_{>21}\right]$ & $6(17.6)$ & $16(11.6)$ & 0.46 & 0.50 & 0.64 & $0.18-2.33$ \\
\hline$\left[\mathrm{AT}_{16-21}\right] /\left[\mathrm{AT}_{16-21}\right]$ & $6(17.6)$ & $29(21.0)$ & 0.05 & 0.82 & 1.16 & $0.33-4.06$ \\
\hline$\left[\mathrm{AT}_{16-21}\right] /\left[\mathrm{AT}_{>21}\right]$ & $6(17.6)$ & $24(17.4)$ & 0.004 & 1.00 & 0.96 & $0.27-3.39$ \\
\hline$\left[\mathrm{AT}_{>21}\right] /\left[\mathrm{AT}_{>21}\right]$ & $0(0.0)$ & $2(1.4)$ & $4.27^{*}$ & $0.04 *$ & - & - \\
\hline Allele $^{d}$ & & & & & & \\
\hline$\left[\mathrm{AT}_{<16}\right]$ & $28(41.2)$ & $108(39.1)$ & & & Referent & \\
\hline$\left[\mathrm{AT}_{16-21}\right]$ & $28(41.2)$ & $124(44.9)$ & 0.22 & 0.64 & 1.15 & $0.64-2.06$ \\
\hline$\left[\mathrm{AT}_{>21}\right]$ & $12(17.6)$ & $44(15.9)$ & 0.02 & 1.00 & 0.95 & $0.44-2.04$ \\
\hline Dominant model & & & & & & \\
\hline$\left[\mathrm{AT}_{<16}\right] /\left[\mathrm{AT}_{16-21}\right]+\left[\mathrm{AT}_{16-21}\right] /\left[\mathrm{AT}_{16-21}\right]+$ & $34(100.0)$ & $136(98.6)$ & $0.04 *$ & $0.85^{*}$ & - & - \\
\hline & $0(0.0)$ & $2(1.4)$ & & & - & - \\
\hline ICOS c.1554_4GT(8_15) & & & & & & \\
\hline Genotype $^{e}$ & & & & & & \\
\hline$(\mathrm{s}) /(\mathrm{s})$ & $0(0.0)$ & $1(0.7)$ & $0.39^{*}$ & $0.53^{*}$ & - & - \\
\hline$(\mathrm{s}) /(\mathrm{m})$ & $0(0.0)$ & $5(3.6)$ & $0.47^{*}$ & $0.49^{*}$ & - & - \\
\hline$(\mathrm{s}) /(1)$ & $3(8.8)$ & $4(2.9)$ & $0.41^{*}$ & $0.52 *$ & 0.42 & $0.09-2.07$ \\
\hline$(\mathrm{m}) /(\mathrm{m})$ & $17(50.0)$ & $54(39.1)$ & & & Referent & \\
\hline$(\mathrm{m}) /(\mathrm{l})$ & $10(29.4)$ & $67(48.6)$ & 2.97 & 0.08 & 2.11 & $0.89-4.98$ \\
\hline (l)/(1) & $4(11.8)$ & $7(5.1)$ & $0.26^{*}$ & $0.61^{*}$ & 0.55 & $0.14-2.11$ \\
\hline Allele $^{f}$ & & & & & & \\
\hline (s) & $3(4.4)$ & $11(4.0)$ & $0.03^{*}$ & $0.85^{*}$ & 0.90 & $0.24-3.35$ \\
\hline (m) & 44 (647.) & $180(65.2)$ & & & Referent & \\
\hline (l) & $21(30.9)$ & $85(30.8)$ & 0.001 & 1.00 & 0.99 & $0.55-1.77$ \\
\hline
\end{tabular}


Table 3 continued

\begin{tabular}{|c|c|c|c|c|c|c|}
\hline & \multicolumn{2}{|c|}{ Familial autoimmune thyroid history } & \multirow[t]{2}{*}{$\chi^{2}$} & \multirow[t]{2}{*}{$p$-value } & \multirow[t]{2}{*}{ Odds ratio } & \multirow{2}{*}{$\begin{array}{l}95 \% \text { confidence } \\
\text { interval }\end{array}$} \\
\hline & $\begin{array}{l}\text { Without } \\
(n=34) n(\%)\end{array}$ & $\begin{array}{l}\text { With } \\
(n=138) n(\%)\end{array}$ & & & & \\
\hline \multicolumn{7}{|l|}{ Dominant model } \\
\hline \multirow[t]{2}{*}{$(\mathrm{s}) /(\mathrm{m})+(\mathrm{m}) /(\mathrm{m})+(\mathrm{m}) /(\mathrm{l}) v s$. others } & $27(79.4)$ & $126(91.3)$ & 3.92 & 0.05 & 2.72 & $0.98-7.55$ \\
\hline & $7(20.6)$ & $12(8.7)$ & & & 0.37 & 0.13-1.02 \\
\hline$C T L A-4 \mathrm{~g} .319 \mathrm{C}>\mathrm{T}$ (rs5742909) & Whole genotype & a & \multicolumn{4}{|c|}{$\chi^{2}=\mathbf{1 6 . 8 9}, \mathrm{df}=2, p=\mathbf{0 . 0 0 0 2}$} \\
\hline$C T L A-4 \mathrm{c} .49 \mathrm{~A}>\mathrm{G}(\mathrm{rs} 231775)$ & Whole genotype & b & \multicolumn{4}{|c|}{$\chi^{2}=2.78, \mathrm{df}=2, p=0.25$} \\
\hline \multirow[t]{2}{*}{ CTLA-4g.*642AT(8_33) } & Whole genotype & c & \multicolumn{4}{|c|}{$\chi^{2}=1.44, \mathrm{df}=5, p=0.92$} \\
\hline & Whole allele & d & \multicolumn{4}{|c|}{$\chi^{2}=0.33, \mathrm{df}=2, p=0.85$} \\
\hline \multirow[t]{2}{*}{ ICOSc.1554+4GT(8_15) } & Whole genotype & e & \multicolumn{4}{|c|}{$\chi^{2}=8.75, \mathrm{df}=5, p=0.12$} \\
\hline & Whole allele & f & \multicolumn{4}{|c|}{$\chi^{2}=0.026678, \mathrm{df}=2, p=0.986749$} \\
\hline
\end{tabular}

Bold indicates statistical significant association

ICOSc.1554+4GT(8_15): (s)-short alleles with 8 and 9 repeats of GT pair; (m)-medium alleles with 10 and 11 repeats of GT pair; (1)-long alleles with 12 or more repeats of GT pair

$\wedge_{-}-p$-value after Bonferroni correction; * $p$-value after Yate's correction; "-global $p$-value for genotype

was observed $\left(\chi_{\text {Global }}^{2}=18.40, p_{\text {corrected }}=0.03\right)$. In particular, haplotypes $\mathrm{CCA}\left[\mathrm{AT}_{<16}\right] \mathrm{AT}(\mathrm{m})$ and $\left.\mathrm{TTA}_{2} \mathrm{AT}_{>21}\right] \mathrm{GG}(\mathrm{m})$ were significantly associated with sporadic GD ( $p=0.055078 ; p=0.003176)$, whereas TCG[AT $16-21] \mathrm{GG}$ (m) was over-represented in patients with familial thyroid autoimmune history $(p=0.025898) \quad$ (Supplementary Table 8).

\section{Response to medical treatment}

Univariate analysis revealed significant associations of two CTLA-4 3'UTR SNPs: CT60 (rs3087243) and Jo31 (rs11571302)-genotypes at both polymorphic sites were differentially distributed with regard to sustained response to medical treatment $(p=0.007 ; p=0.03)$. Both, the CT60 (rs3087243) [AA] and Jo31 (rs11571302) [TT] homozygosity significantly increased the response rate $\left(p_{\text {corrected }}\right.$ $=0.007, \mathrm{OR}=0.16,95 \% \mathrm{CI}: 0.04-0.60 ; p_{\text {corrected }}=0.01$, $\mathrm{OR}=0.30,95 \% \mathrm{CI}: 0.12-0.79)$. This was also confirmed at the allele level $(p=0.002 ; p=0.008)$, and lack of those alleles statistically significantly reduces the success of medical treatment $(p=0.01, \quad \mathrm{OR}=2.24,95 \% \quad \mathrm{CI}$ : $1.21-4.15 ; \quad p=0.02, \quad \mathrm{OR}=2.02, \quad 95 \% \quad \mathrm{CI}: 1.10-3.89)$ (Table 4 and Supplementary Table 9).

Haplotype TTA[AT $\left.{ }_{16-21}\right] \mathrm{GT}(\mathrm{l})$ significantly reduced, whereas $\mathrm{TCA}\left[\mathrm{AT}_{<16}\right] \mathrm{AT}(\mathrm{m})$ increased the success of anti-thyroid treatment $(p=0.008184, \mathrm{OR}=9.048,95 \%$ CI: $1.287-63.593 ; \quad p=0.007347, \quad$ OR $=0.438, \quad 95 \%$ CI: 0.238-0.806, Supplementary Table 10).

\section{Response to medical treatment-multivariate associations}

Finally, unconditional stepwise multivariate logistic regression analysis of all studied polymorphisms, including three prone genetic parameters: presence of $\mathrm{G}$ allele at CT60 (rs3087243) (genotype [GG] and [GA]), Jo31 (rs11571302) [GG] genotype, and presence of $C T L A-4 \mathrm{~g}$. *642AT(8_33)[AT AT-21 $\left._{16}\right]$ allele, indicated only the CT60 (rs3087243) marker as an independent disease relapse factor $\left(\chi^{2}=114.6, p=0.009\right)$. СT60 (rs3087243) [G] allele 4.51fold decreased success of anti-thyroid treatment $(p=0.02$, $95 \%$ CI: $1.24-16.3$ ).

\section{CD28/CTLA-4/ICOS gene polymorphisms and GO}

\section{The course of $G O$ - univariate associations}

The CTLA-4g.*642AT(8_33)[AT $\left.{ }_{16-21}\right]$ homozygosity increased the risk of GO 2.42-fold $(p=0.004,95 \%$ CI: 1.31-4.46). Moreover, the same genotype was associated with GO severity $(p=0.0001, \mathrm{OR}=4.55,95 \%$ CI: 2.01-10.31; $p=0.01, \mathrm{OR}=3.91,95 \%$ CI: $1.33-11.49)$, but favored the inactive outcome $(p=0.004, \mathrm{OR}=3.37$, 95\% CI: 1.44-7.89). The same observation was true for allele (Table 5, Supplementary Tables 11 and 12).

Concerning CT60 (rs3087243), the [AA] genotype significantly reduced the risk of GO and heavy GO outcome: severe eye symptoms as well as active GO course $(p=0.02$, $\mathrm{OR}=0.48,95 \%$ CI: $0.26-0.90 ; p=0.01, \mathrm{OR}=0.35,95 \%$ CI: $0.15-0.83 ; p=0.02, \mathrm{OR}=0.35,95 \% \mathrm{CI}: 0.14-0.86)$, which was also true for presence of $[\mathrm{A}]$ allele $(p=0.02$, OR $=0.64,95 \%$ CI: $0.43-0.94 ; p=0.02, \mathrm{OR}=0.55,95 \% \mathrm{CI}$ : $0.34-0.90 ; \quad p=0.05, \quad \mathrm{OR}=0.60,95 \% \quad \mathrm{CI}: \quad 0.36-1.00)$ (Table 5, Supplementary Tables 11 and 12).

Although the association of CTLA-4g.319C $>\mathrm{T}$ (rs5742909) with GO development was observed only at the allele level $(p=0.04)$ (Table 5), this SNP was associated with GO outcome (Supplementary Table 11). The rare [T] 
Table 4 CTLA-4g.*642AT(8_32), CT60 (rs3087243, CTLA-4g.*6230G>A), Jo31 (CTLA-4g.*10223G>T) genotype and allele frequencies in GD patients with respect to response to anti-thyroid treatment

\begin{tabular}{|c|c|c|c|c|c|c|}
\hline & \multicolumn{2}{|c|}{ Anti-thyroid treatment } & \multirow[t]{2}{*}{$\chi^{2}$} & \multirow[t]{2}{*}{$p$-value } & \multirow[t]{2}{*}{ Odds ratio } & \multirow{2}{*}{$\begin{array}{l}95 \% \text { confidence } \\
\text { interval }\end{array}$} \\
\hline & $\begin{array}{l}\text { Response } \\
n(\%)\end{array}$ & $\begin{array}{l}\text { No response } \\
n(\%)\end{array}$ & & & & \\
\hline \multicolumn{7}{|l|}{ CTLA-4g.*642AT(8_33) } \\
\hline \multicolumn{7}{|l|}{ Genotype $^{c}$} \\
\hline$\left[\mathrm{AT}_{<16}\right] /\left[\mathrm{AT}_{<16}\right]$ & $22(22.9)$ & $9(11.8)$ & & & Referent & \\
\hline$\left[\mathrm{AT}_{<16}\right] /\left[\mathrm{AT}_{16-21}\right]$ & $28(29.2)$ & $24(31.6)$ & 2.38 & 0.12 & 2.10 & $0.81-5.41$ \\
\hline$\left[\mathrm{AT}_{<16}\right] /\left[\mathrm{AT}_{>21}\right]$ & $13(13.5)$ & $9(11.8)$ & 0.81 & 0.37 & 1.69 & $0.54-5.35$ \\
\hline$\left[\mathrm{AT}_{16-21}\right] /\left[\mathrm{AT}_{16-21}\right]$ & $20(20.8)$ & 15 (19.7) & 1.36 & 0.24 & 1.83 & $0.66-5.11$ \\
\hline$\left[\mathrm{AT}_{16-21}\right] /\left[\mathrm{AT}_{>21}\right]$ & $13(13.5)$ & $17(22.4)$ & 4.76 & 0.03 & 3.20 & $1.11-9.22$ \\
\hline$\left[\mathrm{AT}_{>21}\right] /\left[\mathrm{AT}_{>21}\right]$ & $0(0.0)$ & $2(2.6)$ & $1.66^{*}$ & $0.20 *$ & - & - \\
\hline \multicolumn{7}{|l|}{ Allele $^{d}$} \\
\hline$\left[\mathrm{AT}_{<16}\right]$ & $85(44.3)$ & $51(33.6)$ & & & Referent & \\
\hline$\left[\mathrm{AT}_{16-21}\right]$ & $81(42.2)$ & $71(46.7)$ & 2.49 & 0.11 & 1.46 & $0.91-2.34$ \\
\hline$\left[\mathrm{AT}_{>21}\right]$ & $26(13.5)$ & $30(19.7)$ & 4.20 & 0.04 & 1.92 & $1.03-3.61$ \\
\hline \multicolumn{7}{|l|}{ Dominant model } \\
\hline \multirow{2}{*}{$\begin{array}{l}{\left[\mathrm{AT}_{<16}\right] /\left[\mathrm{AT}_{16-21}\right]+\left[\mathrm{AT}_{16-21}\right] /\left[\mathrm{AT}_{16-21}\right]+} \\
{\left[\mathrm{AT}_{<16}\right] /\left[\mathrm{AT}_{>21}\right] \text { vs. others }}\end{array}$} & $61(63.5)$ & $56(73.7)$ & 2.01 & 0.16 & 1.61 & $0.83-3.10$ \\
\hline & $35(36.5)$ & $20(26.3)$ & & & 0.62 & $0.32-1.20$ \\
\hline \multicolumn{7}{|l|}{ CT60(CTLA-4)(g.*6230G > A, rs3087243) } \\
\hline \multicolumn{7}{|l|}{ Genotype $^{e}$} \\
\hline [GG] & $33(34.4)$ & $41(53.9)$ & & & Referent & \\
\hline$[\mathrm{GA}]$ & $48(50.0)$ & $32(42.1)$ & 3.66 & 0.06 & 0.54 & $0.28-1.02$ \\
\hline$[\mathrm{AA}]$ & 15 (15.6) & $3(3.9)$ & $7.22 *$ & $0.007 *$ & 0.16 & $0.04-0.60$ \\
\hline \multicolumn{7}{|l|}{ Allele } \\
\hline$[\mathrm{G}]$ & $114(59.4)$ & $114(75.0)$ & 9.27 & 0.002 & 2.05 & $1.29-3.27$ \\
\hline$[\mathrm{A}]$ & $78(40.6)$ & $38(25.0)$ & & & 0.49 & $0.31-0.78$ \\
\hline \multicolumn{7}{|l|}{ Dominant model } \\
\hline \multirow[t]{2}{*}[\mathrm{GG}]{$+[\mathrm{GA}] v s .[\mathrm{AA}]$} & $81(84.4)$ & $73(96.1)$ & 4.99* & $0.03^{*}$ & 4.51 & $1.25-16.20$ \\
\hline & 15 (15.6) & $3(3.9)$ & & & 0.22 & $0.06-0.80$ \\
\hline \multicolumn{7}{|l|}{ Recessive model } \\
\hline \multirow[t]{2}{*}[\mathrm{AA}]{$+[\mathrm{GA}]$ vs. $[\mathrm{GG}]$} & $63(63.6)$ & $35(46.1)$ & 6.63 & 0.01 & 0.45 & $0.24-0.83$ \\
\hline & $33(34.4)$ & $41(53.9)$ & & & 2.24 & $1.21-4.15$ \\
\hline \multicolumn{7}{|l|}{ Jo31 (CTLA-4)(g.*10223G>T, rs11571302) } \\
\hline \multicolumn{7}{|l|}{ Genotype $^{f}$} \\
\hline [GG] & $28(29.2)$ & $35(46.1)$ & & & Referent & \\
\hline$[\mathrm{GT}]$ & $47(49.0)$ & $33(43.4)$ & 2.89 & 0.09 & 0.56 & $0.29-1.10$ \\
\hline$[\mathrm{TT}]$ & $21(21.9)$ & $8(10.5)$ & 6.24 & 0.01 & 0.30 & $0.12-0.79$ \\
\hline \multicolumn{7}{|l|}{ Allele } \\
\hline$[\mathrm{G}]$ & $103(53.6)$ & $103(67.8)$ & 7.04 & 0.008 & 1.82 & $1.17-2.83$ \\
\hline$[\mathrm{T}]$ & $89(46.4)$ & $49(32.2)$ & & & 0.55 & $0.35-0.86$ \\
\hline \multicolumn{7}{|l|}{ Dominant model } \\
\hline$[\mathrm{GG}]+[\mathrm{GT}] v s .[\mathrm{TT}]$ & $75(78.1)$ & $68(89.5)$ & 3.90 & 0.05 & 2.38 & $0.99-3.73$ \\
\hline & $21(21.9)$ & $8(10.5)$ & & & 0.42 & $0.17-1.01$ \\
\hline Recessive model & & & & & & \\
\hline$[\mathrm{TT}]+[\mathrm{GT}]$ vs. $[\mathrm{GG}]$ & $68(70.8)$ & $41(53.9)$ & 5.21 & 0.02 & 0.48 & $0.26-0.91$ \\
\hline & $28(29.2)$ & $35(46.1)$ & & & 2.07 & $1.10-3.89$ \\
\hline CTLA-4g.*642AT(8_33) & & Whole genoty & & c & $\chi^{2}=7.52, \mathrm{~d}$ & $p=0.19$ \\
\hline & & Whole allele & & d & $\chi^{2}=4.86, \mathrm{~d}$ & $p=0.09$ \\
\hline CT60 (CTLA-4g.*6230G >A, rs3087243) & & Whole genoty & & e & $\chi^{2}=9.87, \mathrm{~d}$ & $p=0.007$ \\
\hline Jo31 (CTLA-4g.*10223G > T, rs11571302) & & Whole genoty & & $\mathbf{f}$ & $\chi^{2}=6.82, d$ & $p=0.03$ \\
\hline
\end{tabular}

Bold indicates statistical significant association

^- $p$-value after Bonferroni correction; *-p-value after Yate's correction; "-global $p$-value for genotype 
Table 5 CTLA-4g.319C>T (rs5742909), CTLA-4g.*642AT(8_32), CT60 (rs3087243, CTLA-4g.*6230G>A) genotype and allele frequencies in GD patients stratified by occurrence of GO and in control group

\begin{tabular}{|c|c|c|c|c|c|c|c|c|c|}
\hline & $\begin{array}{l}\text { Control } \\
\text { group I } n(\%)\end{array}$ & $\begin{array}{l}\text { GD patients } \\
\text { without } \\
\text { GO II } \\
n(\%)\end{array}$ & $\begin{array}{l}\text { GD patients } \\
\text { with GO III } \\
n(\%)\end{array}$ & $p_{\text {global }}^{\#}$ & & $\chi^{2}$ & $p$ & Odds ratio & $\begin{array}{l}95 \% \\
\text { confidence } \\
\text { interval }\end{array}$ \\
\hline \multicolumn{10}{|c|}{$C T L A-4 \mathrm{~g} .319 \mathrm{C}>\mathrm{T}(\mathrm{rs} 5742909)$} \\
\hline \multicolumn{10}{|c|}{ Genotype } \\
\hline$[\mathrm{CC}]$ & $309(80.3)$ & $23(82.1)$ & $105(72.9)$ & & Referent & & & & \\
\hline \multirow[t]{3}{*}[\mathrm{CT}]{} & $74(19.2)$ & $4(14.3)$ & $36(25.0)$ & & I:II & 0.89 & 0.35 & 0.65 & $0.26-1.60$ \\
\hline & & & & & I:III & 2.40 & 0.12 & 1.43 & $0.91-2.23$ \\
\hline & & & & & II:III & $0.91 *$ & $0.34 *$ & 1.97 & $0.64-6.09$ \\
\hline \multirow[t]{3}{*}[\mathrm{TT}]{} & $2(0.5)$ & $1(3.6)$ & $3(2.1)$ & & $\mathrm{I}: \mathrm{II}$ & $0.60 *$ & 0.44 & 8.13 & $0.71-93.74$ \\
\hline & & & & & I:III & $1.55^{*}$ & $0.21 *$ & 4.41 & $0.73-26.78$ \\
\hline & & & & & II:III & $0.09 *$ & $0.76^{*}$ & 0.66 & $0.07-6.61$ \\
\hline \multicolumn{10}{|l|}{ Allele } \\
\hline \multirow[t]{3}{*}[\mathrm{C}]{} & $692(89.9)$ & $50(89.3)$ & $246(85.4)$ & & I:II & 2.28 & 0.13 & 0.56 & $0.27-1.20$ \\
\hline & & & & & I:III & 4.13 & 0.04 & 0.66 & $0.44-0.99$ \\
\hline & & & & & II:III & 0.58 & 0.44 & 0.70 & $0.28-1.74$ \\
\hline \multirow[t]{3}{*}[\mathrm{T}]{} & $78(10.1)$ & $6(10.7)$ & $42(14.6)$ & & I:II & 2.28 & 0.13 & 1.77 & $0.84-3.77$ \\
\hline & & & & & I:III & 4.13 & 0.04 & 1.51 & $1.01-2.27$ \\
\hline & & & & & II:III & 0.58 & 0.44 & 1.42 & $0.57-3.53$ \\
\hline \multicolumn{10}{|l|}{ Dominant model } \\
\hline \multirow[t]{6}{*}[\mathrm{CC}]{$+[\mathrm{CT}]$ vs. $[\mathrm{TT}]$} & $383(99.5)$ & $27(96.4)$ & $141(97.9)$ & & I:II & $0.47 *$ & $0.49 *$ & 0.14 & $0.01-1.61$ \\
\hline & & & & & I:III & $1.32 *$ & $0.25 *$ & 0.25 & $0.04-1.48$ \\
\hline & & & & & II:III & $0.04 *$ & $0.84 *$ & 1.74 & $0.17-17.37$ \\
\hline & $2(0.5)$ & $1(3.6)$ & $3(2.1)$ & & I:II & $0.47 *$ & $0.49 *$ & 7.09 & $0.62-80.72$ \\
\hline & & & & & I:III & $1.32 *$ & $0.25^{*}$ & 4.07 & $0.67-24.64$ \\
\hline & & & & & II:III & $0.04 *$ & $0.84 *$ & 0.57 & $0.06-5.73$ \\
\hline \multicolumn{10}{|l|}{ Recessive model } \\
\hline \multirow[t]{6}{*}[\mathrm{TT}]{$+[\mathrm{CT}]$ vs. $[\mathrm{CC}]$} & $76(19.7)$ & $5(17.9)$ & $39(24.8)$ & & I:II & $0.00 *$ & $1.00 *$ & 0.88 & $0.33-2.40$ \\
\hline & & & & & I:III & $3.32 *$ & $0.07 *$ & 0.66 & $0.42-1.03$ \\
\hline & & & & & II:III & $0.62 *$ & $0.43 *$ & 1.71 & $0.61-4.81$ \\
\hline & $309(80.3)$ & $23(82.1)$ & $105(72.9)$ & & I:II & $0.00 *$ & $1.00 *$ & 1.13 & $0.42-3.07$ \\
\hline & & & & & I:III & $3.32 *$ & $0.07 *$ & 1.51 & $0.97-2.36$ \\
\hline & & & & & II:III & $0.62 *$ & $0.43 *$ & 0.59 & $0.21-1.65$ \\
\hline \multicolumn{10}{|l|}{ CTLA-4g.*642AT(8_33) } \\
\hline \multicolumn{10}{|l|}{ Genotype $^{c}$} \\
\hline$\left[\mathrm{AT}_{<16}\right] /\left[\mathrm{AT}_{<16}\right]$ & $99(25.4)$ & $4(14.3)$ & $27(18.8)$ & & Referent & & & & \\
\hline \multirow[t]{3}{*}[\mathrm{AT}_{<16}]{$/\left[\mathrm{AT}_{16-21}\right]$} & $104(26.7)$ & $12(42.9)$ & $40(27.8)$ & & I:II & $2.48 *$ & $0.12 *$ & 2.86 & $0.89-9.15$ \\
\hline & & & & & I:III & 1.45 & 0.23 & 1.41 & $0.81-2.47$ \\
\hline & & & & & II:III & $0.72 *$ & $0.40 *$ & 0.49 & $0.14-1.69$ \\
\hline \multirow[t]{3}{*}[\mathrm{AT}_{<16}]{$/\left[\mathrm{AT}_{>21}\right]$} & $64(16.5)$ & $2(7.1)$ & $20(13.9)$ & & I:II & $0.02 *$ & $1.00 *$ & 0.77 & $0.14-4.35$ \\
\hline & & & & & I:III & 0.16 & 0.69 & 1.15 & $0.59-2.21$ \\
\hline & & & & & II:III & $0.00 *$ & $1.00 *$ & 1.48 & $0.25-8.90$ \\
\hline \multirow[t]{3}{*}[\mathrm{AT}_{16-21}]{$/\left[\mathrm{AT}_{16-21}\right]$} & $50(12.9)$ & $2(7.1)$ & $33(22.9)$ & & I:II & $0.19 *$ & $0.67 *$ & 0.99 & $0.18-5.59$ \\
\hline & & & & & I:III & 8.22 & 0.004 & 2.42 & $1.31-4.46$ \\
\hline & & & & & II:III & $0.34 *$ & $0.56^{*}$ & 2.44 & $0.42-14.38$ \\
\hline$\left[\mathrm{AT}_{16-21}\right] /\left[\mathrm{AT}_{>21}\right]$ & $54(13.9)$ & $6(21.4)$ & $24(16.7)$ & & $\mathrm{I}: \mathrm{II}$ & $1.52 *$ & $0.22 *$ & 2.75 & $0.74-10.17$ \\
\hline & & & & & I:III & 2.24 & 0.13 & 1.63 & $0.86-3.10$ \\
\hline & & & & & II:III & $0.16^{*}$ & $0.69 *$ & 0.59 & $0.15-2.35$ \\
\hline$\left[\mathrm{AT}_{>21}\right] /\left[\mathrm{AT}_{>21}\right]$ & $18(4.6)$ & $2(7.1)$ & $0(0.0)$ & & I:II & $0.35^{*}$ & $0.55^{*}$ & 2.75 & $0.47-16.15$ \\
\hline & & & & & I:III & $3.45^{*}$ & $0.06^{*}$ & - & - \\
\hline & & & & & II:III & $4.62 *$ & $0.03 *$ & - & - \\
\hline Allele ${ }^{d}$ & & & & & & & & & \\
\hline$\left[\mathrm{AT}_{<16}\right]$ & $366(47.0)$ & $22(39.3)$ & $114(39.6)$ & ns & Referent & & & & \\
\hline$\left[\mathrm{AT}_{16-21}\right]$ & $258(33.2)$ & $22(39.3)$ & $130(45.1)$ & & I:II & 1.26 & 0.26 & 1.42 & $0.77-2.62$ \\
\hline & & & & & I:III & 10.10 & 0.001 & 1.62 & $1.20-2.18$ \\
\hline & & & & & II:III & 0.16 & 0.69 & 1.14 & $0.60-2.17$ \\
\hline$\left[\mathrm{AT}_{>21}\right]$ & $154(19.8)$ & $12(21.4)$ & $44(15.3)$ & & I:II & 0.49 & 0.48 & 1.30 & $0.63-2.69$ \\
\hline & & & & & I:III & 0.18 & 0.67 & 0.92 & $0.62-1.36$ \\
\hline & & & & & II:III & 0.75 & 0.39 & 0.71 & $0.32-1.55$ \\
\hline
\end{tabular}


Table 5 continued

\begin{tabular}{|c|c|c|c|c|c|c|c|c|c|}
\hline & $\begin{array}{l}\text { Control } \\
\text { group I } n(\%)\end{array}$ & $\begin{array}{l}\text { GD patients } \\
\text { without } \\
\text { GO II } \\
n(\%)\end{array}$ & $\begin{array}{l}\text { GD patients } \\
\text { with GO III } \\
n(\%)\end{array}$ & $p_{\text {global }}{ }^{\#}$ & & $\chi^{2}$ & $p$ & Odds ratio & $\begin{array}{l}95 \% \\
\text { confidence } \\
\text { interval }\end{array}$ \\
\hline \multicolumn{10}{|l|}{ Dominant model } \\
\hline \multirow{6}{*}{$\begin{array}{c}{\left[\mathrm{AT}_{<16}\right] /\left[\mathrm{AT}_{16-21}\right]+} \\
{\left[\mathrm{AT}_{<<66}\right] /\left[\mathrm{AT}_{>21}\right]+\left[\mathrm{AT}_{16-21}\right] /} \\
{\left[\mathrm{AT}_{16-21}\right] v s . \text { others }}\end{array}$} & $203(53.6)$ & $20(71.4)$ & $97(67.4)$ & & I:II & 3.36 & 0.07 & 2.17 & $0.93-5.04$ \\
\hline & & & & & I:III & 8.12 & 0.004 & 1.79 & $1.20-2.68$ \\
\hline & & & & & II:III & 0.18 & 0.67 & 0.83 & $0.34-2.01$ \\
\hline & $176(46.4)$ & $8(28.6)$ & 47 (32.6) & & I:II & 3.36 & 0.07 & 0.46 & $0.20-1.07$ \\
\hline & & & & & I:III & 8.12 & 0.004 & 0.56 & $0.37-0.84$ \\
\hline & & & & & II:III & 0.18 & 0.67 & 1.21 & $0.50-2.95$ \\
\hline \multicolumn{10}{|c|}{ CT60 (CTLA-4)(g.*6230G >A, rs3087243) } \\
\hline \multicolumn{10}{|c|}{ Genotype $^{e}$} \\
\hline [GG] & $133(34.3)$ & $9(32.1)$ & $65(45.1)$ & & Referent & & & & \\
\hline \multirow[t]{3}{*}[\mathrm{GA}]{} & $187(48.2)$ & $17(60.7)$ & $63(43.8)$ & & I:II & 0.48 & 0.49 & 1.34 & $0.58-3.11$ \\
\hline & & & & & I:III & 2.79 & 0.09 & 0.69 & $0.46-1.04$ \\
\hline & & & & & II:III & 2.23 & 0.13 & 0.51 & $0.21-1.24$ \\
\hline \multirow[t]{3}{*}[\mathrm{AA}]{} & $68(17.5)$ & $2(7.1)$ & $16(11.1)$ & & I:II & $0.56^{*}$ & $0.46 *$ & 0.43 & $0.09-2.07$ \\
\hline & & & & & I:III & 5.47 & 0.02 & 0.48 & $0.26-0.90$ \\
\hline & & & & & II:III & $0.08 *$ & $0.78 *$ & 1.11 & $0.22-5.64$ \\
\hline \multicolumn{10}{|l|}{ Allele } \\
\hline \multirow[t]{3}{*}[\mathrm{G}]{} & $453(58.4)$ & $35(62.5)$ & $193(67.0)$ & & I:II & 0.37 & 0.55 & 1.19 & $0.68-2.08$ \\
\hline & & & & & I:III & 6.57 & 0.01 & 1.45 & $1.09-1.93$ \\
\hline & & & & & II:III & 0.43 & 0.51 & 1.22 & $0.67-2.21$ \\
\hline \multirow[t]{3}{*}[\mathrm{A}]{} & $323(41.6)$ & $21(37.5)$ & $95(33.0)$ & & I:II & 0.37 & 0.55 & 0.84 & $0.48-1.47$ \\
\hline & & & & & I:III & 6.57 & 0.01 & 0.69 & $0.52-0.92$ \\
\hline & & & & & II:III & 0.43 & 0.51 & 0.82 & $0.45-1.49$ \\
\hline \multicolumn{10}{|l|}{ Dominant model } \\
\hline \multirow[t]{6}{*}[\mathrm{GG}]{$+[\mathrm{GA}] v s .[\mathrm{AA}]$} & $320(82.5)$ & $26(92.9)$ & $128(88.9)$ & & I:II & $1.34 *$ & $0.25 *$ & 2.76 & $0.64-11.92$ \\
\hline & & & & & I:III & 3.25 & 0.07 & 1.70 & $0.95-3.04$ \\
\hline & & & & & II:III & $0.08 *$ & $0.77 *$ & 0.62 & $0.13-2.84$ \\
\hline & $68(17.5)$ & $2(7.1)$ & $16(11.1)$ & & I:II & $1.34 *$ & $0.25^{*}$ & 0.36 & $0.08-1.56$ \\
\hline & & & & & I:III & 3.25 & 0.07 & 0.59 & $0.33-1.05$ \\
\hline & & & & & II:III & $0.08 *$ & $0.77 *$ & 1.63 & $0.35-7.50$ \\
\hline \multicolumn{10}{|l|}{ Recessive model } \\
\hline \multirow[t]{6}{*}[\mathrm{AA}]{$+[\mathrm{GA}] v s .[\mathrm{GG}]$} & $255(65.7)$ & 19 (67.9) & 79 (54.9) & & I:II & 0.06 & 0.80 & 1.11 & $0.49-2.52$ \\
\hline & & & & & I:III & 5.14 & 0.02 & 0.64 & $0.43-0.94$ \\
\hline & & & & & II:III & 1.62 & 0.20 & 0.58 & $0.24-1.36$ \\
\hline & $134(34.4)$ & $9(32.1)$ & $65(45.1)$ & & I:II & 0.06 & 0.80 & 0.90 & $0.40-2.05$ \\
\hline & & & & & I:III & 5.14 & 0.02 & 1.57 & $1.06-2.31$ \\
\hline & & & & & II:III & 1.62 & 0.20 & 1.74 & $0.74-4.10$ \\
\hline
\end{tabular}

Bold indicates statistical significant association

$C T L A-4$ g. 319C $>$ T (rs5742909): GD patients without GO vs. control group: $\chi^{2}=3.70, \mathrm{df}=2, p=0.16$; GD patients with GO vs. control group: $\chi^{2}$ $=5.12, \mathrm{df}=2, p=0.08$; GD patients without GO vs. GD patients with GO: $\chi^{2}=1.65, \mathrm{df}=2, p=0.44$

CTLA-4g.*642AT(8_32): Genotype: GD patients without GO vs. control group: $\chi^{2}=7.25, \mathrm{df}=5, p=0.20$; GD patients with GO vs. control group: $\chi^{2}=\mathbf{1 6 . 5 3}, \mathrm{df}=\mathbf{5}, p=\mathbf{0 . 0 0 6}$; GD patients without GO vs. GD patients with $\mathrm{GO}: \chi^{2}=\mathbf{1 6 . 3 1}, \mathrm{df}=\mathbf{5}, p=\mathbf{0 . 0 0 6}$

CTLA-4g.*642AT(8_32): Allele: GD patients without GO vs. control group: $\chi^{2}=1.33, \mathrm{df}=2, p=0.51$; GD patients with GO vs. control group: $\chi^{2}$ $=\mathbf{1 3 . 1 9}, \mathrm{df}=\mathbf{2}, p=\mathbf{0 . 0 0 1}$; GD patients without GO vs. GD patients with $\mathrm{GO}: \chi^{2}=1.45, \mathrm{df}=2, p=0.48$

CT60 (CTLA-4g.*6230G >A, rs3087243): GD patients without GO vs. control group: $\chi^{2}=2.54, \mathrm{df}=2, p=0.28$; GD patients with GO vs. control group: $\chi^{2}=\mathbf{6 . 5 1}, \mathrm{df}=\mathbf{2}, p=\mathbf{0 . 0 4}$; GD patients without GO vs. GD patients with GO: $\chi^{2}=2.72, \mathrm{df}=2, p=0.26$

OR odds ratio, $95 \%$ CI $95 \%$ confidence intervals

$\wedge$ - $p$-value after Bonferroni correction; * $p$-value after Yate's correction

allele was associated with active $\mathrm{GO}(p=0.002$, $\mathrm{OR}=2.08$, $95 \% \quad$ CI: $1.29-3.37 ; \quad p=0.04, \quad$ OR $=2.05, \quad 95 \%$ CI: $1.04-4.40)$. A more evident association was observed when the presence of this allele was considered $(p=0.002$, $\mathrm{OR}=2.35,95 \%$ CI: $1.36-4.05 ; p=0.01, \mathrm{OR}=2.67,95 \%$ CI: 1.24-5.76) (Supplementary Table 12). 
Albeit the CTLA-4c.49A $>\mathrm{G}$ (rs231775) and Jo31 (rs11571302) SNPs were not related to GO development, they were associated with the GO outcome (Supplementary Table 12). The CTLA-4c.49A> [G] allele favored severe disease outcome $(p=0.04, \quad \mathrm{OR}=1.63, \quad 95 \% \quad \mathrm{CI}$ : 1.01-2.61), which was confirmed when the recessive model $([\mathrm{GG}]+[\mathrm{AG}] v s .[\mathrm{AA}])$ was adopted $(p=0.03$, OR $=1.89$, $95 \%$ CI: $1.06-3.36 ; p=0.007, \quad \mathrm{OR}=2.70,95 \%$ CI: 1.30-5.61) (Supplementary Table 12).

When GO is present, carriers of Jo31 (rs11571302) [TT] genotype were protected against severe eye symptoms $(p=0.04, \mathrm{OR}=0.37,95 \%$ CI: $0.14-0.96)$, which was confirmed in a dominant model $([\mathrm{GG}]+[\mathrm{GT}]$ vs. [TT]) $(p=0.04, \mathrm{OR}=0.61,95 \%$ CI: $0.38-0.98 ; p=0.05, \mathrm{OR}=$ $0.43,95 \%$ CI: 0.18-1.02) (Supplementary Table 12).

\section{The course of GO-haplotype associations}

A statistically significant difference in haplotype distribution between GD patients regarding GO occurrence and healthy controls was observed (Supplemental Table 13). When severity and activity status were taken into account, different global haplotypes distribution was seen in case of absence of GO symptoms compared to severity and activity status, but they did not reach statistical significance (in both $\left.p_{\text {corrected }}=0.06\right)($ Supplemental Table 14). Haplotype TCG $\left[\mathrm{AT}_{16-21}\right] \mathrm{GG}(\mathrm{m})$ favors not only direct $\mathrm{GO}$ development, but also the severe and active GO course $(p=0.015201$; $p=0.015448 ; p=0.015266$ ) (Supplementary Tables 13 and 14).

Heavy outcome of GO were also promoted by TCG $\left.\left[\mathrm{AT}_{16-21}\right] \mathrm{GG}(\mathrm{m}), \mathrm{TCA} \mathrm{AT}_{<16}\right] \mathrm{GT}(\mathrm{m})$, and TCA[AT $\left.\mathrm{AT}_{>21}\right] \mathrm{AT}$ (m) haplotypes (Supplemental Table 14), while haplotypes CCG[AT $\left.{ }_{16-21}\right] \mathrm{GG}(\mathrm{m}), \quad \mathrm{TCG}\left[\mathrm{AT}_{>21}\right] \mathrm{GG}(\mathrm{m}), \quad$ TCG[AT $\left.\mathrm{A}_{<16}\right]$ GG(l), TCA[AT $\left.<16] \mathrm{GG}(\mathrm{m}), \quad \mathrm{TCA}^{\mathrm{A}} \mathrm{AT}_{<16}\right] \mathrm{GT}(\mathrm{l}), \quad$ and $\mathrm{TCA}\left[\mathrm{AT}_{16-21}\right] \mathrm{GG}(\mathrm{m})$ were linked to non-severe mild $\mathrm{GO}$ outcome. TCA[AT $\left.\mathrm{A}_{<16}\right] \mathrm{AT}(\mathrm{l})$ haplotype protected against an active course of eye changes (Supplementary Table 14).

In contrast, haplotypes $\mathrm{TCA}\left[\mathrm{AT}_{>21}\right] \mathrm{GG}(\mathrm{m})$ and $\mathrm{TCG}$ $\left[\mathrm{AT}_{<16}\right] \mathrm{GG}(\mathrm{m})$ directly favored light $\mathrm{GO}$ outcome without clinical eye symptoms $(p=0.000174 ; p=0.015662)$ (Supplementary Table 13), which were also confirmed within GO stratified according to activity and severity ( $p=0.038863 ; p=0.030938 ; p=0.012530)$ (Supplementary Table 14).

Haplotype TCG[AT $\left.\mathrm{AT}_{>21}\right] \mathrm{GG}(\mathrm{m})$ was statistically significantly more frequent in healthy persons $(p=0.003529$; $p=0.029963$ ) (Supplementary Tables 13 and 14).

\section{The course of $G O$-multivariate associations}

The unconditional multivariate logistic regression analysis of all studied polymorphisms, including four prone genetic parameters: presence of $[\mathrm{G}]$ allele at $C T L A-4 \mathrm{c} .49 \mathrm{~A}>\mathrm{G}$ (rs231775) (genotype [GG] and [AG]), presence of [G] allele at CT60 (rs3087243) (genotype [GG] and [GA]), presence of $C T L A-4 \mathrm{~g} . * 642 \mathrm{AT}\left(8 \_33\right)\left[\mathrm{AT}_{16-21}\right]$ allele, Jo31 (rs11571302) [GG] genotype and environmental factors: smoking and male gender, showed that presence of CTLA4g. $* 642 \mathrm{AT}\left(8 \_33\right)\left[\mathrm{AT}_{16-21}\right]$ allele $(p=0.04, \mathrm{OR}=2.10$, $95 \%$ CI: $1.03-4.27)$ and male gender $(p=0.00008$, $\mathrm{OR}=4.29,95 \% \mathrm{CI}: 2.11-8.72)$ were independent risk factors for GO $\left(\chi_{2}^{2}=24.1, p=0.00001\right)$.

\section{Discussion}

Since a number of studies have established a central role for $\mathrm{T}$ cells in GD [2, 5-7], the region containing genes encoding the immune regulatory molecules, CTLA-4, CD28, and ICOS, is good candidate for disease risk factor. Selected for our study polymorphic sites located within genes encoded, these co-stimulatory molecules had to have been reported to be associated with altered immune response and/or susceptibility to autoimmune disease $[9,13$, 18-22, 24-26]. Based on the fact that all three genes are close to one another within the chromosomal region $2 \mathrm{q} 33$, and that LD was observed between polymorphisms located within these genes, we postulated that the association between GD (and/or its clinical phenotype) and this region might represent the effects of a combination of polymorphic variations of these three genes. We therefore looked for a possible association of studied polymorphisms as one susceptible genetic region defined as a haplotype block with the GD phenotype.

Our study indicated specific haplotypes significantly linked with sporadic GD and haplotypes over-represented in patients with a familial history of thyroid disease. These results led us to the conclusion that patients with sporadic incidence and those with a familial background of GD are characterized by a combination of various markers, and it is therefore possible that they are genetically different. These markers may be predictive factors for the development of GD in families in whom thyroid disease is observed. Although the $2 \mathrm{q} 33$ chromosomal region is linked to GD, the association between the two was found to be different in female and male subjects. Our group of patients was too small for stratification by gender at the haplotype level in the context of GO outcome, therefore the possibility that differences in our overall population at the univariate level will also be true in GO subgroups cannot be excluded. To date, no literature concerning such a relationship has been published. In terms of predicting the clinical outcome, two specific haplotypes are possible: one reducing (TTA[AT $\left.\left.{ }_{16-21}\right] \mathrm{GT}(\mathrm{l})\right)$ and another $\left.\left(\mathrm{TCA}^{\mathrm{A}} \mathrm{AT}_{<16}\right] \mathrm{AT}(\mathrm{m})\right)$ increasing the successful outcome of anti-thyroid treatment. 
The roles of genetic factors in the GO pathomechanism are not well characterized. Although a genetic predisposition to GD has been confirmed, to increase one's risk of developing the disease, the significance of genotype-phenotype correlations in GD remains speculative, and the results of family-based studies are conflicting. So far-and only at a univariate levelpolymorphisms located in CTLA-4 have been linked to GO risk and outcome predictors. Therefore, we tested the combined impact of the 2q33 region and GO course, and observed statistically significantly different haplotype associations in GO status. In our search for deeper insight, specifically GO course (severity and activity), the differences were more evident. Particularly, one specific haplotype TCG[AT $\left.{ }_{16-21}\right] \mathrm{GG}(\mathrm{m})$ favored not only the development of GO but also a severe outcome. The haplotypes $\mathrm{TCA}\left[\mathrm{AT}_{>21}\right] \mathrm{GG}(\mathrm{m})$ and $\mathrm{TCG}\left[\mathrm{AT}_{<16}\right] \mathrm{GG}(\mathrm{m})$, on the other hand, were directly linked to GD without any eye symptoms. As this study is the first association analysis based on the aforementioned concept, our observations require confirmation by future studies. It must be noted that we were able to collect a relatively large cohort of patients with severe eye symptoms and compare them with subjects with the milder form of GO.

When we used the classical approach-a univariate association model - the association with GD in our southern Polish population was seen only in case of two polymorphisms located within the CTLA-4 3'UTR region: CT60 (rs3087243) and g.*642AT(8_33). When we looked deeper, taking ancestral background into account, three CTLA-4 polymorphic variants (g.319C $>\mathrm{T}$ (rs5742909) [CC], c. $49 \mathrm{~A}>\mathrm{G} \quad(\mathrm{rs} 231775) \quad[\mathrm{G}], \quad \mathrm{g} . * 642 \mathrm{AT}\left(8 \_33\right)\left[\mathrm{AT}_{>21}\right] /$ $\left.\left[\mathrm{AT}_{>21}\right]\right)$ were associated with the family burden of GD. This finding may provide some evidence in support of our hypothesis; specifically that the superiority of the association defined as one susceptible locus expressed as the haplotype over the individual polymorphism association. As compared to other studies, which describe only univariate analyses [28, 29, 30, 31], our research takes a deeper and more detailed approach. The most GD-essential genetic marker in our study, CTLA-4g.*642AT(8_33), was also relevant in other populations [2, 18, 25]. Importantly, this marker has functional implications; specifically the long AT-repeat allele is associated with reduced control of T-cell proliferation and thus contributes to the pathogenesis of GD [25].

Other Polish studies [29, 30, 31] focused mostly on CTLA-4c.49A $>\mathrm{G}$ (rs231775) and revealed a significant association of this marker with disease; however, we did not. On the other hand, the association of the marker CT60 (rs3087243) observed in our group was not as significant as in another study of Polish patients with GD [29]. Our observation of a significant association of the CT60 (rs3087243) [G] allele aligns with the results of other reports $[9,18,28]$, including a large meta-analysis where this polymorphic site strongly increased GD risk [28].

Since the goal of anti-thyroid drug therapy (to restore an euthyroid state) may be achieved in up to $50 \%$ of GD patients, defining the genetic predictors of remission could be helpful in clinical practice; therefore, we tested studied markers to forecast the clinical outcome. At a univariate level, CT60 (rs3087243) [AA] and Jo31 (rs11571302) [TT] homozygosity significantly increased the success of antithyroid treatment, but multivariate logistic regression analysis only pointed CT60 (rs3087243) as an independent risk factor for disease progression.

Previous studies focusing on the CTLA-4c.49A $>\mathrm{G}$ (rs231775) polymorphism [32, 33, 34, 35] showed its association with a lower chance of remission after discontinuation of propylthiouracil treatment [32, 33, 34], indicating that GD patients with the $C T L A-4 \mathrm{c} .49 \mathrm{~A}>\mathrm{G}$ (rs231775) [GG] genotype may not be the best candidates for anti-thyroid drug therapy. Moreover, GD patients with the [G] allele at this SNP needed to continue anti-thyroid drug treatment for longer periods to achieve remission, indicating that this polymorphic variant may be an indicator of poor prognosis [33, 34]. In contrast, but in line with our results, Kim et al. [35] did not observe such a relationship when it came to CTLA-4g.319C > T (rs5742909) and CTLA$4 \mathrm{c} .49 \mathrm{~A}>\mathrm{G}$ (rs231775). So far, no research has assessed the impact of CD28 and ICOS variation on the success of medical treatment, and only variation within CTLA-4 has been documented to be a prognostic factor for remission of disease after treatment. The observed association is probably due to the functional consequence of studied polymorphic markers.

We found an association between CTLA-4 polymorphic variations and the development and course of GO on a univariate level. If severity of GO was considered an outcome, associations were observed in the case of markers for CTLA-4c.49A >G (rs231775), CTLA-4g.*642AT(8_33), CT60 (rs3087243), and Jo31 (rs11571302). When looking for a combined effect (genetics and environment), CTLA4g.*642AT(8_33) and male gender were found to be independent risk factors for GO. CTLA-4c.49A $>\mathrm{G}$ (rs231775) was linked to GO course but not development. A large meta-analysis [37] of inconsistent results of studies, focusing on the association between CTLA-4 gene polymorphisms and GO, revealed no evidence in support of an association between this SNP and eye changes.

Moreover, in the one-marker association analysis no relationship between studied polymorphisms within $C D 28$ and ICOS and GD or the course of the disease was observed in the overall group association analysis. Our results confirmed previous data showing a lack of association between these polymorphic sites and GD [9, 27]. The absence of a 
relationship between the $C D 28 \mathrm{c} .17+3 \mathrm{~T}>\mathrm{C}$ (rs3116496) SNP and the disease was evidenced by the lack of impact on thyroid antibody production [35]; however, stratification by gender showed that females with long alleles at the ICOSc.1554+4GT(8_15) polymorphic site were more prone to GD. This is the first gender-stratified analysis; therefore, corroboration by future studies is necessary.

$\mathrm{We}$, and others, have provided evidence to support the idea that, in a single gene polymorphic variation, changes within CTLA-4 may be considered to be GD risk factors and factors capable of modifying the clinical course of the disease. We also postulated that because of the location-in one common chromosomal region-and $\mathrm{LD}$, the analyzed CD28/CTLA-4/ICOS gene polymorphic variability should be considered at the haplotype level, rather than by the single-marker model. Moreover, since GD and GO are associated with gender and familial autoimmune thyroid history, these factors should also be taken into account when a patient's genetic background is analyzed.

Acknowledgments The authors would like to thank Proper Medical Writing Sp. $\mathrm{z}$ o. o. for assistance in the final preparation of the manuscript.

Funding The study was supported by funds from the Polish Scientific Research Committee (No. 2 P05B 055 29).

\section{Compliance with ethical standards}

Conflict of interest The authors declare that they have no conflict of interest.

\section{References}

1. P. Caturegli, H. Kimura, R. Rocchi, N.R. Rose, Autoimmune thyroid diseases. Curr. Opin. Rheumatol. 19, 44-48 (2007). doi:10.1097/BOR.0b01e3280113d1a

2. Y. Ban, Y. Tomer, The contribution of immune regulatory and thyroid specific genes to the etiology of Graves' and Hashimoto's diseases. Autoimmunity 36(6-7), 367-379 (2003). doi:10.1080/ 08916930310001603037

3. H.J. Lee, C.W. Li, S.S. Hammerstad, M. Stefan, Y. Tomer, Immunogenetics of autoimmune thyroid diseases: a comprehensive review. J. Autoimmun. 64, 82-90 (2015). doi:10.1016/j. jaut.2015.07.009

4. B.S. Prabhakar, R.S. Bahn, T.J. Smith, Current perspective on the pathogenesis of Graves' disease and ophthalmopathy. Endocr. Rev. 24(6), 802-835 (2003). doi:10.1210/er.2002-0020

5. C. Salamaco, D. Olive, G. Pesce, M. Bagnasco, Costimulatory molecules and autoimmune thyroid diseases. Autoimmunity 35, 159-167 (2002). doi:10.1080/08916930290013441

6. A. Bossowski, A. Stasiak-Barmuta, M. Urban, Relationship between CTLA-4 and CD28 molecule expression on T lymphocytes and stimulating and blocking autoantibodies to the TSHreceptor in children with Graves' disease. Horm. Res. 64, 189-197 (2005). doi:10.1159/000088875

7. N. Matsuoka, K. Eguchi, A. Kawakami, M. Tsuboi, H. Nakamura, H. Kimura, N. Ishikawa, K. Ito, S. Nagataki, Lack of B7-1/BB1 and B7-2/B70 expression on thyrocytes of patients with Graves' disease. Delivery of costimulatory signals from bystander professional antigen-presenting cells. J. Clin. Endocrinol. Metab. 81, 4137-4143 (1996). doi:10.1210/jcem.81.11.8923872

8. T. Kouki, Y. Sawai, C.A. Gardine, M.E. Fisfalen, M.L. Alegre, L.J. DeGroot, CTLA-4 gene polymorphism at position 49 in exon 1 reduces the inhibitory function of CTLA- 4 and contributes to the pathogenesis of Graves' disease. J. Immunol. 165, 6606-6011 (2000). doi:10.4049/jimmunol.165.11.6606

9. Y. Ban, E.S. Concepcion, R.B. Villanueva, D.A. Greenberg, T.F. Davies, Y. Tomer, Analysis of immune regulatory genes in familial and sporadic Graves' disease. J. Clin. Endocrinol. Metab. 89, 4562-4568 (2004). doi:10.1210/jc.2003-031693

10. M. Mäurer, S. Loserth, A. Kolb-Mäurer, A. Ponath, S. Wiese, N. Kruse, P. Rieckmann, A polymorphism in the human cytotoxic T-lymphocyte antigen 4 (CTLA4) gene (exon $1+49)$ alters T-cell activation. Immunogenetics 54, 1-8 (2002). doi:10.1007/s00251002-0429-9

11. S. Anjos, A. Nguyen, H. Ounissi-Benkalha, M.C. Tessier, C. Polychronakos, A common autoimmunity predisposing signal peptide variant of the cytotoxic T-lymphocyte antigen 4 results in inefficient glycosylation of the susceptibility allele. J. Biol. Chem. 277, 46478-46486 (2002). doi:10.1074/jbc.M206894200

12. Y. Xu, P.N. Graves, Y. Tomer, T.F. Davies, CTLA-4 and autoimmune thyroid disease: lack of influence of the A49G signal peptide polymorphism on functional recombinant CTLA-4. Cell. Immunol. 215, 133-140 (2002). doi:10.1016/S0008-8749(02) 00018-7

13. A. Pérez-García, R. de la Cámara, J. Román-Gómez, A. JiménezVelasco, M. Encuentra, J.B. Nieto, J. de la Rubia, A. UrbanoIspizúa, S. Brunet, A. Iriondo, M. González, D. Serrano, I. Espigado, C. Solano, J.M. Ribera, J.M. Pujal, M. Hoyos, D. Gallardo, GVHD/immunotherapy committee of the Spanish group of hematopoietic stem cell transplantation. CTLA-4 polymorphisms and clinical outcome after allogeneic stem cell transplantation from HLA-identical sibling donors. Blood 110(1), 461-467 (2007). doi:10.1182/blood-2007-01-069781

14. A. Ligers, N. Teleshova, T. Masterman, W.X. Huang, J. Hillert, CTLA-4 gene expression is influenced by promoter and exon 1 polymorphisms. Genes Immun. 2, 145-152 (2001)

15. X.B. Wang, X. Zhao, R. Giscombe, A.K. Lefvert, A CTLA-4 gene polymorphism at position -319 in the promoter region affects the expression of protein. Genes Immun. 3, 233-234 (2002). doi:10.1038/sj.gene.6363869

16. D.A. Chistiakov, K.V. Savost'anov, R.I. Turakulov, I.A. Efremov, L.M. Demurov, Genetic analysis and functional evaluation of the $\mathrm{C} / \mathrm{T}(-319)$ and $\mathrm{A} / \mathrm{G}(-1661)$ polymorphisms of the CTLA-4 gene in patients affected with Graves' disease. Clin. Immunol. 118, 233-242 (2006). doi:10.1016/j.clim.2005.09.017

17. S.M. Anjos, M.C. Tessier, C. Polychronakos, Association of the cytotoxic $\mathrm{T}$ lymphocyte-associated antigen 4 gene with type 1 diabetes: evidence for independent effects of two polymorphisms on the same haplotype block. J. Clin. Endocrinol. Metab. 89, 6257-6265 (2004). doi:10.1210/jc.2004-0881

18. H. Ueda, J.M.M. Howson, L. Esposito, J. Heward, H. Snook, G. Chamberlain, D.B. Rainbow, K.M.D. Hunter, A.N. Smith, G. Di Genova, M.H. Herr, I. Dahlman, F. Payne, D. Smyth, C. Lowe, R. C.J. Twells, S. Howlett, B. Healy, S. Nutland, H.E. Rance, V. Everett, L.J. Smink, A.C. Lam, H.J. Cordell, N.M. Walker, C. Bordin, J. Hulme, C. Motzo, F. Cucca, J.F. Hess, M.L. Metzker, J. Rogers, S. Gregory, A. Allahabadia, R. Nithiyananthan, E. Tuomilehto-Wolf, J. Tuomilehto, P. Bingley, K. M. Gillespie, D.E. Undlien, K.S. Rønningen, C. Guja, C. IonescuTrrgoviste, D.A. Savage, A.P. Maxwell, D.J. Carson, C.C. Patterson, J.A. Franklyn, D.G. Clayton, L.B. Peterson, L.S. Wicker, J.A. Todd, S.C.L. Gough, Association of the T-cell 
regulatory gene CTLA4 with susceptibility to autoimmune disease. Nature 423(29), 506-511 (2003)

19. S.M. Anjos, W. Shao, L. Marchand, C. Polychronakos, Allelic effects on gene regulation at the autoimmunity-predisposing CTLA4 locus: a re-evaluation of the $3^{\prime}+6230 \mathrm{G}>\mathrm{A}$ polymorphism. Genes Immun. 6, 305-311 (2005). doi:10.1038/sj. gene.6364211

20. S. Purohit, R. Podolsky, C. Collins, W. Zheng, D. Schatz, A. Muir, D. Hopkins, Y.H. Huang, J.X. She, Lack of correlation between the levels of soluble cytotoxic T-lymphocyte associated antigen-4 (CTLA-4) and the CT-60 genotypes. J. Autoimm. Dis. 2, 8 (2005). doi:10.1186/1740-2557-2-8

21. S. Mayans, K. Lackovic, C. Nyholm, P. Lindgren, K. Ruikka, M. Eliasson, C.M. Cilio, D. Holmberg, CT60 genotype does not affect CTLA-4 isoform expression despite association to T1D and AITD in northern Sweden. BMC Med. Genet. 8, 3 (2007). doi:10.1186/1471-2350-8-3

22. X.B. Wang, M. Kakoulidou, R. Giscombe, Q. Qiu, D. Huang, R. Pirskanen, A.K. Lefvert, Abnormal expression of CTLA-4 by T cells from patients with myasthenia gravis: effect of an AT-rich gene sequence. J. Neuroimmunol. 130, 224-232 (2002). doi:10.1016/S0165-5728(02)00228-X

23. S.M. Anjos, C. Polychronakos, Functional evaluation of the autoimmunity-associated CTLA4 gene: the effect of the (AT) repeat in the 3'untranslated region (UTR). J. Autoimmun. 27, 105-109 (2006). doi:10.1016/j.jaut.2006.06.004

24. L. Malquori, L. Carsetti, G. Ruberti, The 3' UTR of the human CTLA4 mRNA can regulate mRNA stability and translational efficiency. Biochim. Biophys. Acta 1779(1), 60-65 (2008). doi:10.1016/j.bbagrm.2007.10.004

25. M. Takara, T. Kouki, L.J. DeGroot, CTLA-4 AT-repeat polymorphism reduces the inhibitory function of CTLA-4 in Graves' disease. Thyroid 13(12), 1083-1089 (2003). doi:10.1089/ 10507250360731479

26. J. Daroszewski, E. Pawlak, L. Karabon, I. Frydecka, A. Jonkisz, M. Slowik, M. Bolanowski, Soluble CTLA-4 receptor an immunological marker of Graves' disease and severity of ophthalmopathy is associated with CTLA-4 Jo31 and CT60 gene polymorphisms. Eur. J. Endocrinol. 161, 787-793 (2009). doi:10.1530/EJE-09-0600

27. E. Pawlak, I. Kochanowska, I. Frydecka, M. Bilińska, The soluble CTLA-4 receptor: a new marker in autoimmune diseases. Arch. Immunol. Ther. Ex. 53, 336-341 (2005)

28. F.K. Kavvoura, T. Akamizu, T. Awata, Y. Ban, D.A. Chistiakov, I. Frydecka, A. Ghaderi, S.C. Gough, Y. Hiromatsu, R. Ploski, P.W. Wang, Y. Ban, T. Bednarczuk, E.I. Chistiakova, M. Chojm, J.M. Heward, H. Hiratani, S.H. Juo, L. Karabon, S. Katayama, S. Kurihara, R.T. Liu, I. Miyake, G.H. Omrani, E. Pawlak, M. Taniyama, T. Tozaki, J.P. Ioannidis, Cytotoxic T-lymphocyte associated antigen 4 gene polymorphisms and autoimmune thyroid disease: a meta-analysis. J. Clin. Endocrinol. Metab. 92, 3162-3170 (2007). doi:10.1210/jc.2007-0147

29. D. Pastuszak-Lewandoska, E. Sewerynek, D. Domańska, A. Gładyś, R. Skrzypczak, E. Brzeziańska, CTLA-4 gene polymorphisms and their influence on predisposition to autoimmune thyroid diseases (Graves' disease and Hashimoto's thyroiditis). Arch. Med. Sci. 8(3), 415-421 (2012). doi:10.5114/ aoms.2012.28593

30. B. Jurecka-Lubieniecka, R. Ploski, D. Kula, A. Krol, T. Bednarczuk, Z. Kolosza, A. Tukiendorf, S. Szpak-Ulczok, A.
Stanjek-Cichoracka, J. Polanska, B. Jarzab, Association between age at diagnosis of Graves' disease and variants in genes involved in immune response. PLoS ONE 8(3), e59349 (2013). doi:10.1371/journal.pone.0059349

31. T. Bednarczuk, Y. Hiromatsu, T. Fukutani, K. Jazdzewski, P. Miskiewicz, M. Osikowska, J. Nauman, Association of cytotoxic T-lymphocyte-associated antigen-4 (CTLA-4) gene polymorphims and non-genetic factors with Graves' ophthalmopathy in European and Japanese population. Eur. J. Endocrinol. 148, 13-18 (2003). doi:10.1530/eje.0.1480013

32. M. Sahin, M.F. Erdogan, G. Erdogan, Cytotoxic T lymphocyteassociated molecule-4 polymorphisms in Turkish Graves' disease patients and association with probability of remission after antithyroid therapy. Eur. J. Int. Med. 16, 352-355 (2005). doi:10.1016/j.ejim.2005.06.007

33. Y. Kinjo, N. Takasu, I. Komiya, T. Tomoyose, M. Takara, T. Kouki, Y. Shimajiri, K. Yabiku, H. Yoshimura, Remission of Graves' hyperthyroidism and A/G polymorphism at position 49 in exon 1 of cytotoxic T lymphocyte-associated molecule-4 gene. J. Clin. Endocrinol. Metab. 87(6), 2593-2596 (2002). doi:10.1210/ jcem.87.6.8612

34. P.W. Wang, I.Y. Chen, S.H. Juo, E. Hsi, R.T. Liu, C.J. Hsieh, Genotype and phenotype predictors of relapse of Graves' disease after antithyroid drug withdrawal. Eur. Thyroid. J. 1(4), 251-258 (2013). doi:10.1159/000342621

35. K.W. Kim, Y.J. Park, T.Y. Kim, D.J. Park, K.S. Park, B.Y. Cho, Susceptible alleles of the CD40 and CTLA-4 genes are not associated with the relapse after antithyroid withdrawal in Graves' disease. Thyroid 17(12), 1229-1234 (2007). doi:10.1089/ thy.2007.0011

36. Y. Tomer, D.A. Greenberg, G. Barbesino, E. Concepcion, T.F. Davies, CTLA-4 and not CD28 is a susceptibility gene for thyroid autoantibody production. J. Clin. Endocrinol. Metab. 86 (4), 1687-1693 (2001). doi:10.1210/jcem.86.4.7372

37. T. Bednarczuk, B. Gopinath, R. Ploski, J.R. Wall, Susceptibility genes in Graves' ophthalmopathy: searching for a needle in a haystack? Clin. Endocrinol. 67, 3-19 (2007). doi:10.1111/j.13652265.2007.02854.x

38. T. Kaartinen, J. Lappalainen, K. Haimila, M. Autero, J. Partanen, Genetic variation in ICOS regulates mRNA levels of ICOS and splicing isoforms of CTLA4. Mol. Immunol. 44(7), 1644-1651 (2007). doi:10.1016/j.molimm.2006.08.010

39. L. Bartalena, L. Baldeschi, A. Dickinson, A. Eckstein, P. KendallTaylor, C. Marcocci, M. Mourits, P. Perros, K. Boboridis, A. Boschi, N. Currò, C. Daumerie, G.J. Kahaly, G.E. Krassas, C.M. Lane, J.H. Lazarus, M. Marinò, M. Nardi, C. Neoh, J. Orgiazzi, S. Pearce, A. Pinchera, S. Pitz, M. Salvi, P. Sivelli, M. Stahl, G. von Arx, W.M. Wiersinga, European group on Graves' orbitopathy (EUGOGO). Consensus statement of the European group on Graves' orbitopathy (EUGOGO) on management of GO. Eur. J. Endocrinol. 158(3), 273-285 (2008). doi:10.1530/EJE-070666

40. K. Suwalska, E. Pawlak, L. Karabon, A. Tomkiewicz, T. Dobosz, D. Urbaniak-Kujda, K. Kuliczkowski, D. Wolowiec, A. Jedynak, I. Frydecka, Association studies of CTLA-4, CD28, and ICOS gene polymorphisms with B-cell chronic lymphocytic leukemia (B-CLL) in the Polish population. Hum. Immunol. 69, 193-201 (2007). doi:10.1016/j.humimm.2008.01.014 